EUROPEAN CENTRAL BANK

WORKING PAPER SERIES

N0. 360 / MAY 2004

OPTIMAL

MONETARY POLICY

RULES FOR THE

EURO AREA: AN

ANALYSIS USING

THE AREA WIDE

MODEL

by Alistair Dieppe, Keith Küster

and Peter McAdam

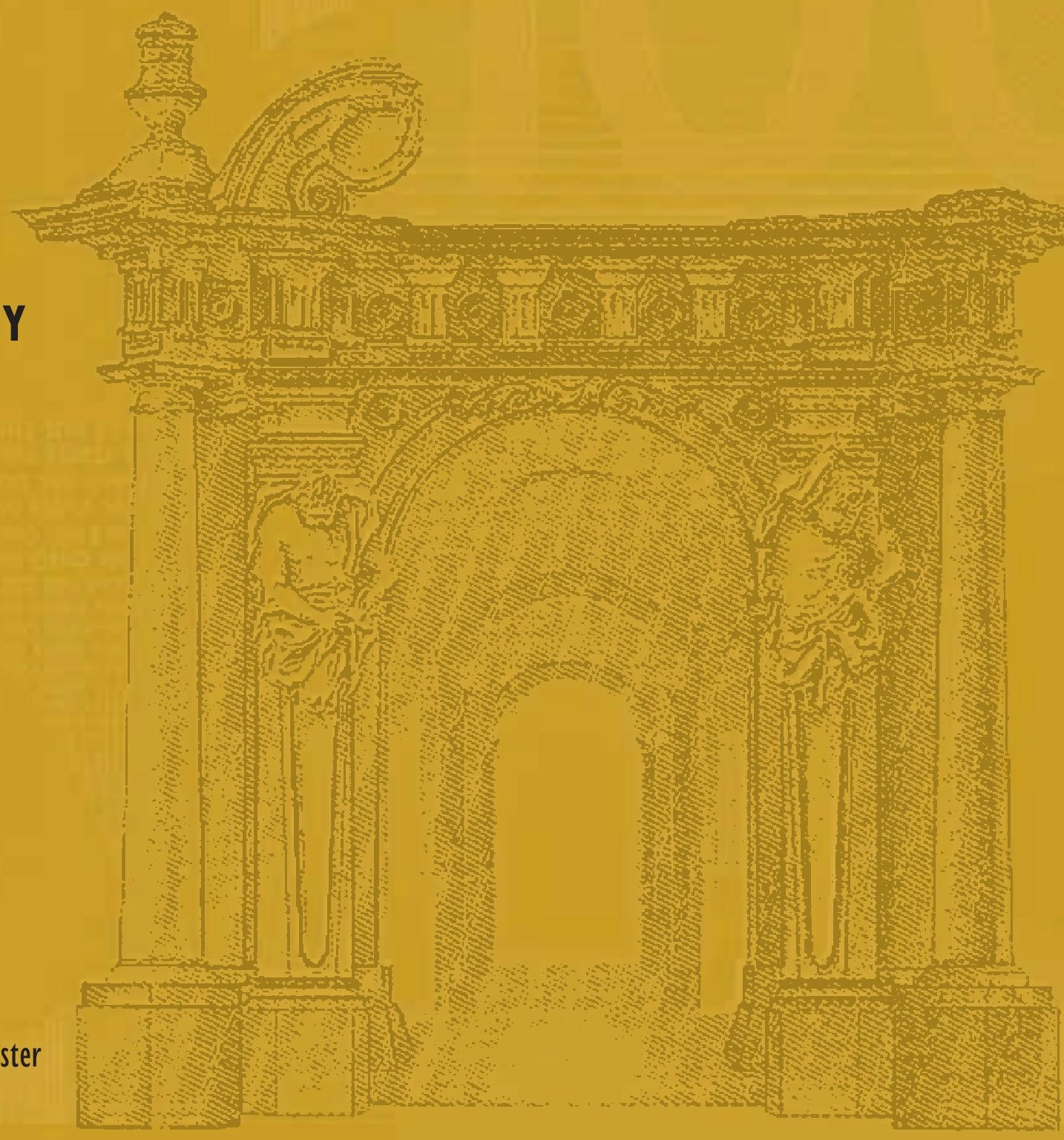




\section{OPTIMAL MONETARY POLICY RULES FOR THE EURO AREA: AN ANALYSIS USING THE AREA WIDE MODEL}

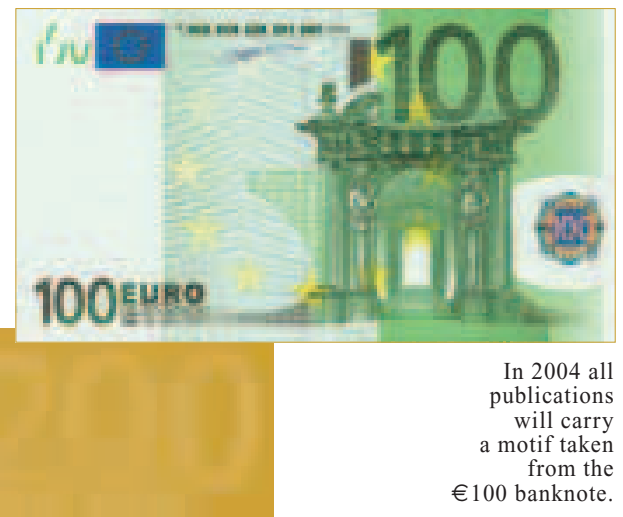

by Alistair Dieppe ${ }^{2}$, Keith Küster ${ }^{3}$ and Peter McAdam ${ }^{2}$

This paper can be downloaded without charge from http://www.ecb.int or from the Social Science Research Network electronic library at http://ssrn.com/abstract_id $=533105$. 


\section{드 European Central Bank, 2004}

\section{Address}

Kaiserstrasse 29

60311 Frankfurt am Main, Germany

Postal address

Postfach 160319

60066 Frankfurt am Main, Germany

Telephone

+496913440

\section{Internet}

http://www.ecb.int

Fax

+496913446000

Telex

411144 ecb d

All rights reserved.

Reproduction for educational and noncommercial purposes is permitted provided that the source is acknowledged.

The views expressed in this paper do not necessarily reflect those of the European Central Bank.

The statement of purpose for the ECB Working Paper Series is available from the ECB website, http://www.ecb.int.

ISSN 1561-0810 (print)

ISSN 1725-2806 (online) 


\section{CONTENTS}

Abstract 4

Non-technical summary 5

1 Introduction 6

2 The modelling framework 8

3 The area wide model ||

3.1 Evaluation of estimated policy rules I3

3.2 The Taylor principle-determinacy regions

4 Optimal policy 19

4.1 Optimized simple rules vs. optimal discretionary policy

4.2 Benchmarking against commitment $\quad 30$

5 Optimal forecast horizons 33

5.1 Optimal forecast horizons for inflation 33

5.2 Optimal forecast horizons for both the output gap and the inflation rate 37

6 Conclusions 38

References 40

A Linearization and parameter choices 43

B First-order form 45

C Empirical variance covariance matrix

D Calculation of optimal policy 46

E Upper bound on $\sigma_{\Delta r}$ for discretion 46

F Optimal commitment 47

G Sensitivity analysis of results 47

European Central Bank working paper series $\quad 50$ 


\begin{abstract}
In this paper, we analyze optimal monetary policy rules in a model of the euro area, namely the ECB's Area Wide Model, which embodies a high degree of intrinsic persistence and a limited role for forward-looking expectations. These features allow us, in large measure, to differentiate our results from many of those prevailing in New Keynesian paradigm models. Specifically, our exercises involve analyzing the performance of various generalized Taylor rules both from the literature and optimized to the reference model. Given the features of our modelling framework, we find that optimal policy smoothing need only be relatively mild. Furthermore, there is substantial gain from implementing forecast-based as opposed to outcome-based policies with the optimal forecast horizon for inflation ranging between two and three years. Benchmarking against fully optimal policies, we further highlight that the gain of additional states in the rule may compensate for a reduction of communicability. Thus, the paper contributes to the debate on optimal monetary policy in the euro area, as well as to the conduct of monetary policy in face of substantial persistence in the transmission mechanism.
\end{abstract}

JEL: E4, E5

Keywords: euro area, monetary policy rule, optimization. 


\section{Non-technical summary}

In this paper, we examine optimal monetary policy in a medium-sized model of the euro area, namely the Area Wide Model (AWM), Fagan et al. (2001). The choice of model is interesting for a number of reasons. First, it contributes to the debate about optimal monetary policy rules for the new euro area; this is a relatively underdeveloped area of research so far. Second, the AWM makes some notable departures from the class of models conventionally studied in the optimal monetary policy literature.

These AWM model features - namely, a high degree of intrinsic persistence and a limited role for forward-looking expectations - allow us, in large measure, to differentiate our results from many of those prevailing in "New-Keynesian" paradigm models (which tend to be highly forward-looking and "micro-founded").

Specifically, our exercises involve analyzing the performance of various generalized Taylor rules both from the literature and optimized to the reference model. Given the features of our modelling framework, we find that optimal policy "smoothing" (i.e. the degree of policy gradualism) need only be relatively mild. Furthermore, there is substantial gain from implementing forecast-based (i.e., forward-looking) as opposed to outcome-based policies (i.e., backward or contemporaneous horizons) with the optimal forecast horizon for inflation ranging between two and three years. Benchmarking simple optimal rules against fully optimal policies, we further highlight that the gain of additional targets in the rule may compensate for a reduction of communicability. That is to say, more complicated rules may significantly outperform relatively simple (and easy to communicate) ones like the Taylor rule. 


\section{Introduction}

In stochastic macro-economic environments subject to nominal rigidities, monetary policy can actively contribute to stabilizing the real and nominal economy. Accordingly, a large body of work has evolved around examining optimal monetary policy rules in sticky-price models. These may be backward-looking as in Svensson (1997) and Ball (1999) or small micro-founded forward-looking models (for early references, Clarida et al., 1999). Such models tend to imply that optimal policy can be written as (or closely approximated by) a Taylor (1993) rule. Often such models feature only a small number of states; consequently, there is only a minor loss in stabilization by limiting feedback to only a subset of such states.

Policy implications for larger scale models, however, have received somewhat less attention - being largely focused on models which assume that agents are substantially forward-looking, as in Levin et al. (1999, 2003). Notwithstanding, the degree to which expectations are in fact forward looking is far from being settled on empirical grounds (e.g., Fuhrer and Estrella, 2002, Fuhrer 1997). In the light of model uncertainty, a prudent policy-maker may therefore feel startled by the strong emphasis on forward-looking expectations combined with small-scale models and in solving this may be unwilling to only resort to small-scale backward-looking models for policy advice.

In this paper, we therefore examine optimal monetary policy in a medium-sized model of the euro area, namely the Area Wide Model (AWM), Fagan et al. (2001). The choice of model is interesting for a number of reasons. First, it contributes to the debate about optimal monetary policy rules for the new euro area; this is a relatively underdeveloped area and therefore of high value added. ${ }^{1}$ Second, the AWM makes some notable departures from the class of models conventionally studied in the optimal monetary policy literature - these include a significant deviation from the strong forward-lookingness and low persistence emphasized in the recent literature as well as from the compact dynamics and limited state variables found in such models. For such reasons, the AWM may be a good candidate for deriving policy implications and benchmarking those against others in the literature.

Defining policy makers' preferences as a weighted average of inflation and output sta-

\footnotetext{
1 The model, for instance, is used extensively in simulation and projection analysis at the ECB. See for example, ECB (2001) and the references in Section 3.
} 
bilization (conditioning on instrument stability), we examine the performance of simple optimized monetary policy rules and fully optimal policy against estimated Taylor rules. In doing so, we also assess the optimal forecast horizon in such monetary policy rules and highlight sources for possible indeterminacy. A key feature of our results is the importance played by intrinsic persistence (i.e., persistence not introduced by monetary policy itself). Coenen (2002), for example, emphasized the risks of underestimating inflation persistence in deriving policy rules. The AWM in fact incorporates substantial intrinsic persistence alongside a sufficiently rich structure and largely backward-looking expectations. Such model differences - from, say, a standard "New-Keynesian" paradigm model - may potentially serve as a robustness check. In particular, we find that - in contrast to many studies, e.g., Levin et al. (2003) - substantial smoothing of interest rates in addition to that induced by persistence of feedback variables is not necessarily a feature of optimal monetary policy. Similarly, given the size of our model we find that the simple optimal rules need not necessarily approximate fully optimal ones (see also, Finan and Tetlow, 1999). Finally, we find that optimal inflation (and output gap) forecast horizons are larger than typically found in the literature owing to the sizeable inflation persistence and the limited degree of forward-lookingness in the model.

Taking these results literally and abstracting from model and policy uncertainty ${ }^{2}$, our analysis suggests that optimal policy should be focused on future states of the economy and incorporate a broad set of information even at the risk of loosing the communicability of policy rules. Indeed, gains from fully optimal discretionary and commitment policy are sizeable due to the persistence of the economy.

The paper proceeds as follows. Section 2 introduces the modelling framework. Section 3 offers a description of the key features of the AWM before section 4 analyzes their implications for the performance of simple optimal rules relative to optimal discretionary policy and more complicated commitment rules. A further section considers the question of the optimal forecast horizon. Section 6 concludes with some policy advice and some considerations of the implications of model uncertainty for the results derived.

\footnotetext{
${ }^{2}$ It should be borne in mind that policy rules of the kind we discuss are to be regarded as broad approximations to actual policy decisions and trade offs. For instance, other than model and parameter uncertainty as mentioned above, we assume rational expectations on behalf of both the central bank and the private sector. Among other things, this amounts to the assumption that the private sector forecast model coincides with the AWM. Another caveat includes the availability of reliable real-time data (e.g., Orphanides, 2001).
} 


\section{The Modelling Framework}

The AWM is not derived explicitly from micro-foundations. We therefore follow the literature in modelling the preferences of a central bank by a conventional quadratic loss function defined in terms of unconditional variances of inflation and the output gap, ${ }^{3}$

$$
L=\min _{r}\{V(\pi)+\lambda V(g a p)+\gamma V(\Delta r)\}
$$

where $V(\cdot)$ marks the unconditional variance of annual(ized) inflation $(\pi)$, the output gap (gap) and interest rate changes $(\Delta r)$, respectively. ${ }^{4}$ While the policymaker's prime mandate is to keep inflation low and stable around a target, he may also have a mandate for stabilizing output around its potential, ${ }^{5}$ the case of flexible inflation targeting in the wording of Svensson (1999). The relative weight of these conflicting goals in the presence of cost-push shocks is captured by the preference parameter $\lambda \in \mathbb{R}_{+} \cdot{ }^{6}$

A typical finding in model-based optimization exercises is that movements in the policy instrument exceed that witnessed in the data. Indeed, as we earlier discussed some degree of policy smoothing is an established empirical regularity. For instance, central banks may be reluctant to change short-term rates frequently in so far as it undermines credibility and inhibits financial-market stability - Cukierman (1990) provides a survey on interestrate smoothing. Uncertainty provides another rationale. Policymakers may be unwilling to completely rely on (model) certainty equivalent policy for pursuing stabilization in the presence of uncertainty about the transmission mechanism, as in Brainard (1967). To capture such features, Svensson (1999) implements a weight, $\gamma>0$, on changes of interest rates directly in the loss-function. Additionally, to derive implications directly linked to the current euro area policy environment, we impose an upper bound on interest

${ }^{3}$ Subsequently, all variables will be in deviations from steady-state values.

${ }^{4}$ Rotemberg and Woodford (1998) and others have shown that similar loss functions can be derived as a second-order approximation to a representative agent's utility function in a simple New-Keynesian model.

${ }^{5}$ Note that here there is no inflation bias.

${ }^{6}$ In a stationary model like ours, this preference function is the limiting case as the policymaker stops discounting of the more standard loss function in curly brackets

$$
L=\lim _{\beta \rightarrow 1}(1-\beta)\left\{\min _{r} E \sum_{j=0}^{\infty} \beta^{j}\left[\pi_{j}^{2}+\lambda g a p_{j}^{2}+\gamma\left(\Delta r_{j}\right)^{2}\right]\right\} .
$$


rate variability of the empirical size while setting $\gamma=0 .{ }^{7}$ As an added benefit, such a constraint precludes zero-bound solutions for the nominal rate.

Svensson (1999a) argues that central banks should follow target rules, assuring the public to enact policy such that it brings internal inflation and possibly output gap forecasts on track with the target(s) in a horizon depending on policy lags. The form of these targeting rules that is communicated to the public is potentially robust to parameter changes since forecasts themselves incorporate all new information. ${ }^{8}$ However, such a strategy may imply rather complex feedback rules, since in principle they would incorporate all the (predetermined) states of the model. Nonetheless, in some cases they may be concisely rewritten in terms of expectations of future inflation and/or the output gap. In general, however, being more complicated, such an instrument rule may be hard to communicate to, and be monitored by, the public. In our view, Svensson's framework might be best described as implementing optimal policy under discretion - we use this as a natural benchmark. Other authors, like Batini and Haldane (1999), understand inflation targeting directly in terms of a simple instrument rule, the most prominent of which may be Taylor's (1993) rule, a generalized version of which is

$$
r_{t}=\rho r_{t-1}+\alpha E_{t} \pi_{t+\theta}+\beta E_{t} g a p_{t+\kappa}
$$

where $\rho \geq 0$ represents the degree of policy smoothing, ${ }^{9}$ expectations are rational, and $(\alpha, \beta) \in \mathbb{R}_{+}^{2}$, with the forecast horizons $(\theta, \kappa) \in \mathbb{Z}^{2} .{ }^{10}$

From an institutional viewpoint, the advantage of simple rules is their transparency

7 We also experimented with a larger upper bound of 2.5 times the empirical upper bound and with implementing a weight on changes of interest rates directly in the loss function. As one could expect, with interest changes being less costly, there is somewhat less smoothing of interest rates and a better stabilization performance. Overall, however, the qualitative results remain unchanged.

8 Nevertheless, changes in the forecasting model of the central bank or parameter uncertainty will inevitably change the way forecasts respond to state variables and thereby they will change the feedback rule of the central bank, i.e. the way interest rates are actually set in response to state variables.

9 Typically $\rho$ is estimated at 0.9 for monthly data (e.g. Clarida et al., 1998). Often 'policy rules' are taken from models featuring multiple interdependent equations in which case strictly speaking there does not exist an identified "monetary policy equation". As an example, even under the plain Taylor rule the first order serial correlation coefficient of interest rates is $\operatorname{Cor}_{1}(r)=0.95$ in the AWM, with $\mathrm{Cor}_{2}(r)=0.89$. Hence, parameter $\rho$ taken per se is a poor guide to any assertion about interest rate smoothing as actually observed, but may rather be understood as a guide to practical policy-making.

10 Approximate and in some cases exact forms of this rule are optimal for a central bank that has a quadratic loss function over inflation and output - as in (1) - in standard macroeconomic environments (e.g., Svensson, 1999, and Ball, 1999). 
and, thus, the ease with which they may be communicated to and be monitored by the outside world. While it is unlikely that any central bank will follow the literal execution of such a rule, they may nonetheless be a good summary guide to rule based policy in general. Furthermore, simple rules (or the implications thereof) are arguably more robust to model mis-specification and uncertainty (see Levin et al., 1999, Levin and Williams, 2003) than policy based on a larger set of states, which might overfit specific model characteristics.

Furthermore, from an empirical point of view, generalized Taylor rules seem to match the data well for the flexible inflation-targeting periods of central banks in Europe and the US. See, for instance, Clarida et al. (1998, 2000), Gerlach and Schnabel (2000) and Gerdesmeier and Roffia (2003). In some cases other variables may enter significantly into the rules, such as exchange rates (e.g., so-called open-economy monetary policy rules), monetary gaps or financial market indicators. Notwithstanding, a principal advantage of even simple forecast-based rules is that a suitable choice of forecast horizon can incorporate contemporaneous and leading-indicator information and, by effectively accounting for policy-transmission lags, induce successfully pre-emptive policy, while lagged or contemporaneous rules necessarily operate at or after the event - an aspect Batini and Haldane (1999) call "lag-encompassing". Second, forecast-based feedback rules are "informationencompassing": if expectations are rational, they will incorporate all states of the economy and make use of all the structure of the economy (though in a restricted way). By choice of optimal feedback horizons, $\theta$ and $\kappa$, the central bank can thus considerably govern how information is implemented into even a simple Taylor rule like (2).

Although the generalized Taylor rule seems to be easily implemented, some caveats emerge. For example, there may be lack of reliable real-time data (see Orphanides, 2001). We thus also employ a Taylor rule feeding back on both, lagged output gaps and inflation to assess the loss involved. Further, Bernanke and Woodford (1997) highlight that for successful implementation of forecast targeting, there appears to be no substitute for explicit structural modelling of the economy and extensive information gathering by the central bank. They illustrate that directly targeting private sector expectations (say, via surveys) may introduce large fluctuations and nullify the information content of private sector forecasts. The borderline at which the increase in complexity due the information encompassing of forecast based rules hinders monitoring is not easily drawn. At some 
point, a discretionary framework might fit the policy framework better. We therefore report results for stabilization using both forecast-based rules and optimal discretionary policy.

Following the literature, in section 4 we analyze the performance of simple rules assuming that due to their simplicity, policymakers could credibly commit themselves to such rules indefinitely. We illustrate that there is a substantial deterioration in stabilization performance involved when contemporaneous data, $\theta=\kappa=0$, is not available or reliable, but lagged data, $\theta=\kappa=-1$, needs to be used to conduct policy. Apart from these outcome-based rules, we illustrate that a common forecast-based rule with a oneyear forecast horizon for inflation, $\theta=4, \kappa=0$, yields substantially better stabilization performance, thereby reaffirming the results of Batini and Haldane (1999). While it is commonly found that the commitment value provided by credible Taylor rules outweighs the loss incurred by conditioning policy only on a subset of information, this ranking depends on model characteristics. The greater is the information content of other variables in the economy (besides the output gap, interest rates and inflation) and the less important is the expectations channel (in the extreme, in a backward-looking model, there is no distinction between Discretion and Commitment), the more valuable will be optimal time-consistent policy as opposed to sub-optimal (optimal simple) policy under commitment.

Optimal discretionary policy in the AWM yields substantially better performance than simple rules unless the central bank feeds back on inflation expectations roughly two years into the future. When we choose the horizon of forecast-based rules optimally (Section 5) we find that these approximate the optimal discretionary stabilization outcome. Due to the more backward-looking structure of the AWM, we demonstrate that if the central bank could tie its hands, conducting relatively complicated rule based commitment policy, it would not do considerably better than under optimal discretionary policy.

\section{The Area Wide Model}

The Area Wide Model by Fagan et al. (2001) is a quarterly estimated structural macroeconomic model that treats the euro area as a single economy. It has a long-run classical equilibrium with a vertical Phillips curve but with some short-run frictions in price/wage 
setting and factor demands. Consequently, activity is demand-determined in the short run but supply-determined in the longer run with employment having converged to a level consistent with the exogenously given level of equilibrium unemployment. Stockflow adjustments are accounted for by, for example, the inclusion of a wealth term in consumption. At present, the treatment of expectations in the model is limited; with the exception of the exchange rate (modelled by forward-looking uncovered interest parity) and the (12-year bond) term structure, the model embodies backward-looking expectations.

The demand channel in the AWM is enacted by short-term interest rates. Long term rates determine government debt payments but do not explicitly enter investment decisions. The expectations channel in principle allows monetary policy to influence inflation via wage and price-setting behavior. In addition to these channels, further channels enter through the exchange rate. Apart from an indirect influence of exchange rates on domestic demand, there is also a direct exchange rate channel to consumer price inflation through the price for imported goods. ${ }^{11}$ In the analysis that follows, one of the key variables is the output gap. This is defined as the ratio of real GDP to Potential Output, which is defined by an aggregate Cobb-Douglas production function with constant returns to scale and neutral technical progress. For this, the trend total factor productivity has been estimated within-sample by applying the Hodrick-Prescott filter to the Solow Residual derived from the production function.

Full model listing and simulation evidence can be found in Fagan et al. (2001) and Dieppe and Henry (2004). Applications of the model include examining the monetary transmission mechanism (McAdam and Morgan, 2003), real exchange rate determination (Detken et al., 2002), unemployment dynamics (Dieppe et al., 2004) and forecasting strategies (McAdam and Mestre, 2003).

In the absence of shocks, the non-stationary system converges to a balanced growth path, on which price ratios are constant and real GDP components grow at a common rate. Log-linearization of models around their steady state values prior to analysis is

${ }^{11}$ Svensson (2000) argues that the direct exchange rate channel invalidates the use of CPI inflation. Similarly, such considerations can be considered in line with the debate in Mankiw and Reis (2003), as to which measure of inflation (i.e., "core" or headline rate) central banks should use in their monetary policy strategies. Since any contribution towards that debate is outside of the scope of this paper, we restrict ourselves to a consumer price inflation measure in order to be comparable to the existing closed economy literature. 
now standard in the literature on optimal and robust policy even for large-scale models (e.g., Levin et al., 1999) and without this step a full-fledged analysis is computationally burdensome. ${ }^{12}$ Appendix A provides details about the linearization.

In order to shed further light on relevant model properties, we next explore the implications of several estimated rules in the AWM.

\subsection{Evaluation of Estimated Policy Rules}

The current model version does not incorporate any estimated rule but rather has been calibrated to the standard Taylor one:

$$
r_{t}=1.5 \pi_{t}+0.5 \operatorname{gap}_{t}
$$

We also report the properties of incorporating three more rules from the literature into the AWM:

$$
\begin{aligned}
& r_{t}=0.87 r_{t-1}+(1-0.87)\left(1.93 \pi_{t}+0.28 \text { gap }_{t}\right), \\
& r_{t}=0.91 r_{t-1}+(1-0.91)\left(1.31 E_{t} \pi_{t+4}+0.25 \text { gap }_{t}\right), \\
& r_{t}=0.18 r_{t-1}+1.51 E_{t} \pi_{t+4}^{4}+0.28 \text { gap }_{t} .
\end{aligned}
$$

Gerdesmeier and Roffia (2003) estimate rule (4) on synthetic euro area data from 1985 to 2002. While we do not believe the ECB is a direct successor of the Bundesbank in all respects, we include rule (5), which Clarida et al. (1998) estimate for Germany from 1979 to 1993, as an additional point of reference. Finally, Gerlach and Schnabel (2000) estimate rule (6) for pooled euro area data from 1990 to 1997. This range thus provides two contemporaneous (outcome-based) and two forward-looking (forecast-based) rules. ${ }^{13}$ Table 1 reports the unconditional standard deviations as implied by the AWM of key variables under these rules.

As can be inferred from the table, the Taylor rule results in large interest changes relative to the data $\left(\sigma_{\Delta r}^{e m p}=0.569\right)$. Some interest-rate smoothing appears therefore to be a necessary feature to match the model to the data. Note that the two rules estimated on synthetic euro data (i.e., rules 4 and 6) fit the historical interest rate volatility quite closely

\footnotetext{
12 McAdam and Hughes Hallett (1999) survey linear and non-linear modelling solution algorithms.

13 Note that rules (4) and (5) were estimated on monthly data. Accordingly, we adapt those rule to the quarterly frequency by setting $\rho_{q}=\rho_{m}^{3}$.
} 
Table 1: Performance of Given Policy Rules ${ }^{a}$

\begin{tabular}{||c||c|c|c|c||}
\hline \hline Rules & $\sigma_{g a p}$ & $\sigma_{\pi_{t}}$ & $\sigma_{\Delta r}$ & $\sigma_{r}$ \\
\hline \hline$(3)$ & 2.028 & 1.229 & 1.530 & 2.327 \\
\hline$(4)$ & 2.406 & 1.359 & 0.681 & 2.444 \\
\hline$(5)$ & 2.295 & 1.390 & 0.389 & 1.702 \\
\hline$(6)$ & 1.896 & 1.177 & 0.626 & 1.852 \\
\hline \hline
\end{tabular}

$a$ For the four different generalized Taylor rules the unconditional standard deviation of the output gap, of annualized quarterly inflation rates, of the annualized interest rate (in percentage terms) and of interest rate changes is reported. The policymaker is assumed to credibly commit to these rules once and forever.

when implemented in the AWM. In light of the stabilization gain comparing forwardlooking policy (6) to the other policy rules, we conclude that "good" policy conduct seems to include smoothing and that there appears to be some leeway for monetary policy in influencing the economy for medium-run stabilization purposes. Supporting this, figures 1 to 4 report impulse responses to a 100 basis point temporary (1 quarter) rise in short-term nominal interest rates for rules (3) to (6).
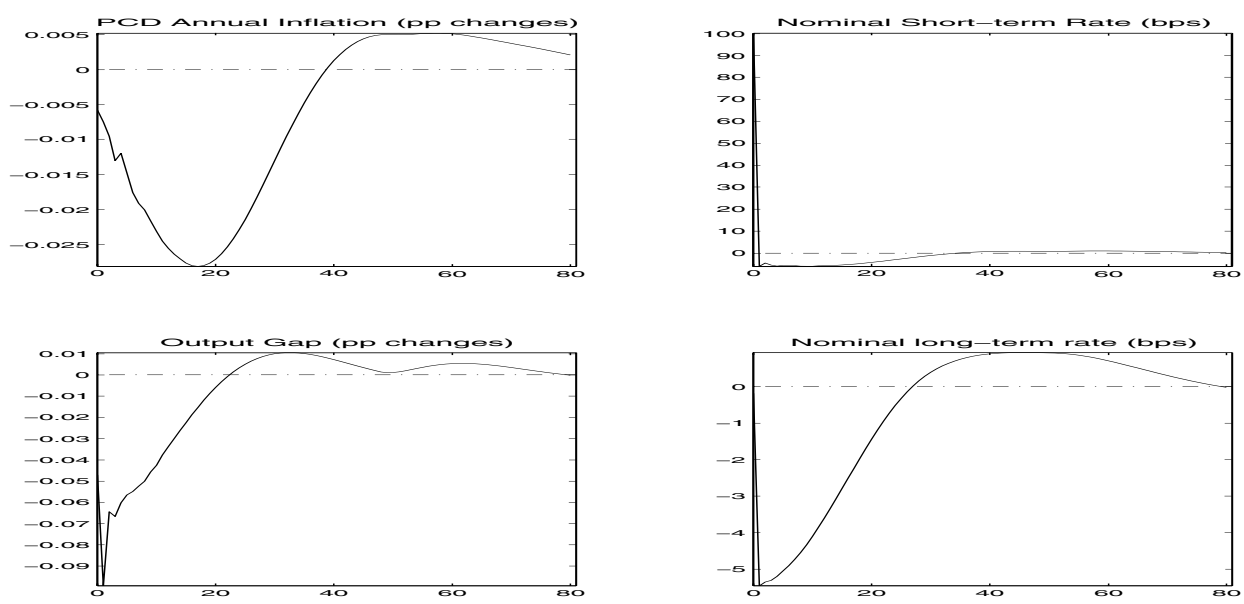

Figure 1: Impulse-responses to a 100bps temporary unanticipated monetary policy shock. The monetary policy rule is the plain Taylor rule $(\rho=0, \alpha=1.5, \beta=0.5)$. All variables are measured in percentage points deviation from steady state.

The plots show that the monetary transmission mechanism in the AWM features 

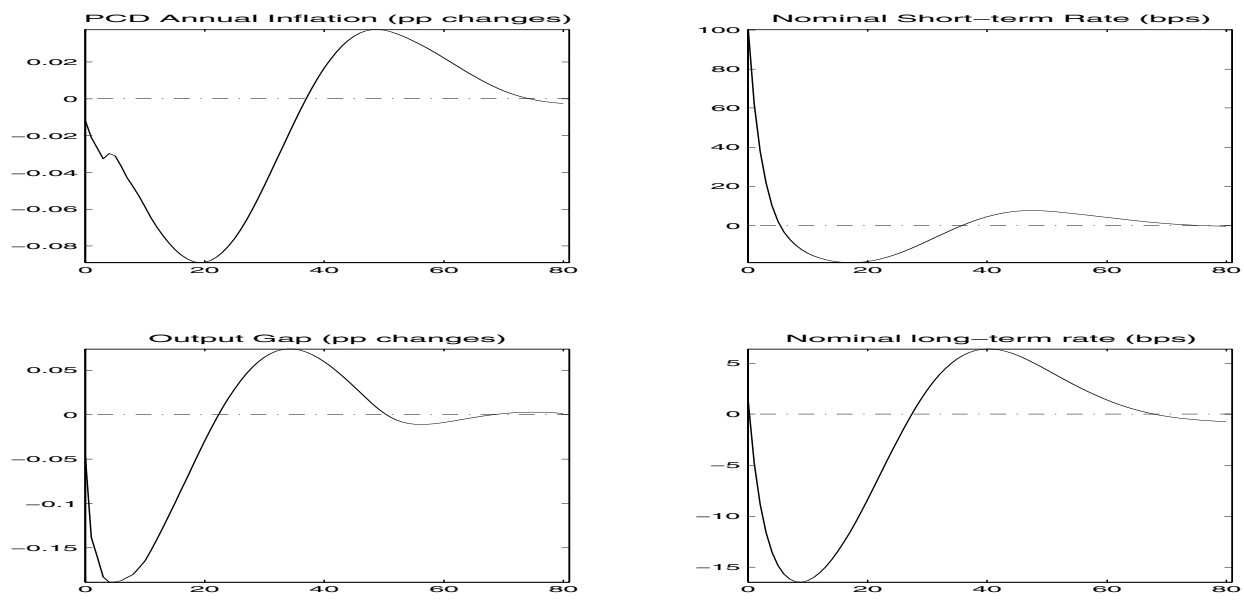

Figure 2: Impulse-responses to a 100bps temporary unanticipated monetary policy shock. The monetary policy rule is the Gerdesmeier and Roffia (2003) rule (4). All variables are measured in percentage points deviation from steady state.

long-lasting effects on output and inflation, with the latter being especially sluggish. In addition, the impulse responses illustrate that a poor conduct of monetary policy could introduce quite pronounced noise at business cycle frequencies. Under the Taylor rule (3), an unanticipated monetary contraction by 100 basis points reduces inflation rates moderately. These reach a trough at a reduction of 0.028 percentage points in inflation more than four years after the shock. Secondary cycles lead to a minor increase in inflation thereafter. The Taylor rule illustrates that while the monetary feedback rule per se does not feature any smoothing, model properties may lead to substantial persistence of shocks. The short-term rate is sharply reduced to a level of $5.98 \mathrm{bps}$ below steady-state in the quarter following the shock, from where it returns only slowly to its steady state value, cycling around steady-state at low frequencies. Output in the short-run is far more responsive than the nominal side, reaching its trough of $0.1 \%$ below steady state in the quarter following the shock. Again, even in the absence of persistent monetary policy per se, model features introduce secondary cycles with output being $0.01 \%$ percent above steady state 8 years after the shock.

Central banks obtain a stronger lever on demand components when committing to more pronounced smoothing. This becomes apparent for the Gerdesmeier and Roffia (2003) rule when implemented in the AWM. Here, the real and nominal sides show stronger amplitudes, in addition to peaking later. The maximum reduction of annual inflation is three times larger than under a plain Taylor rule, with the peak response being half a 

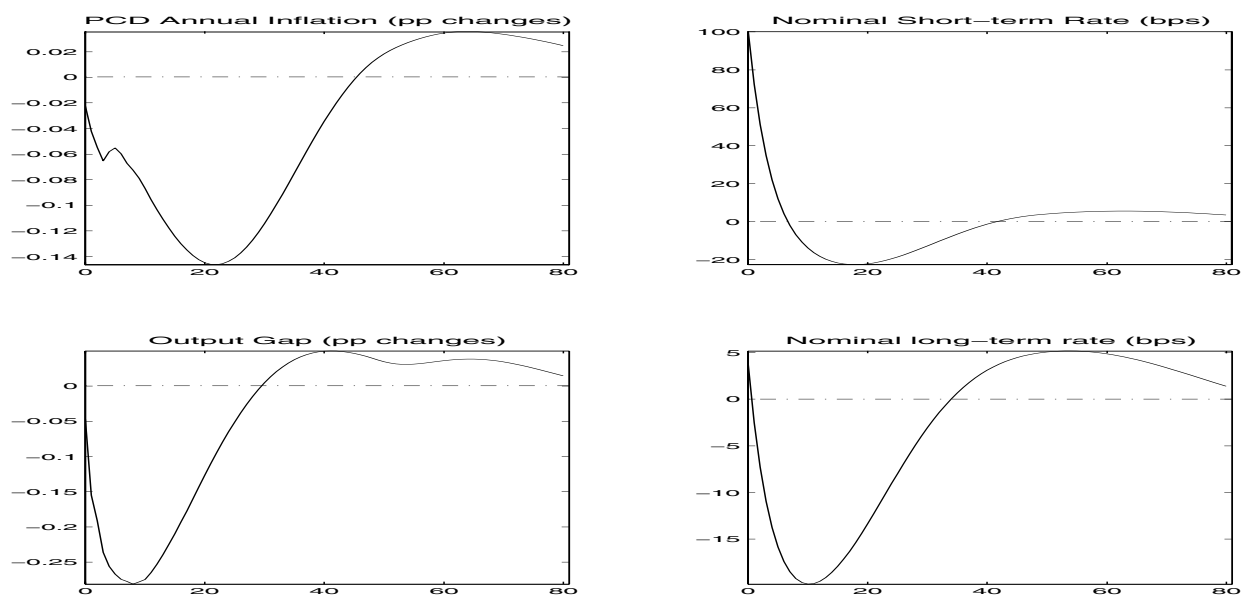

Figure 3: Impulse-responses to a 100bps temporary unanticipated monetary policy shock. The monetary policy rule is the Clarida et al. (1998) rule (5). All variables are measured in percentage points deviation from steady state.
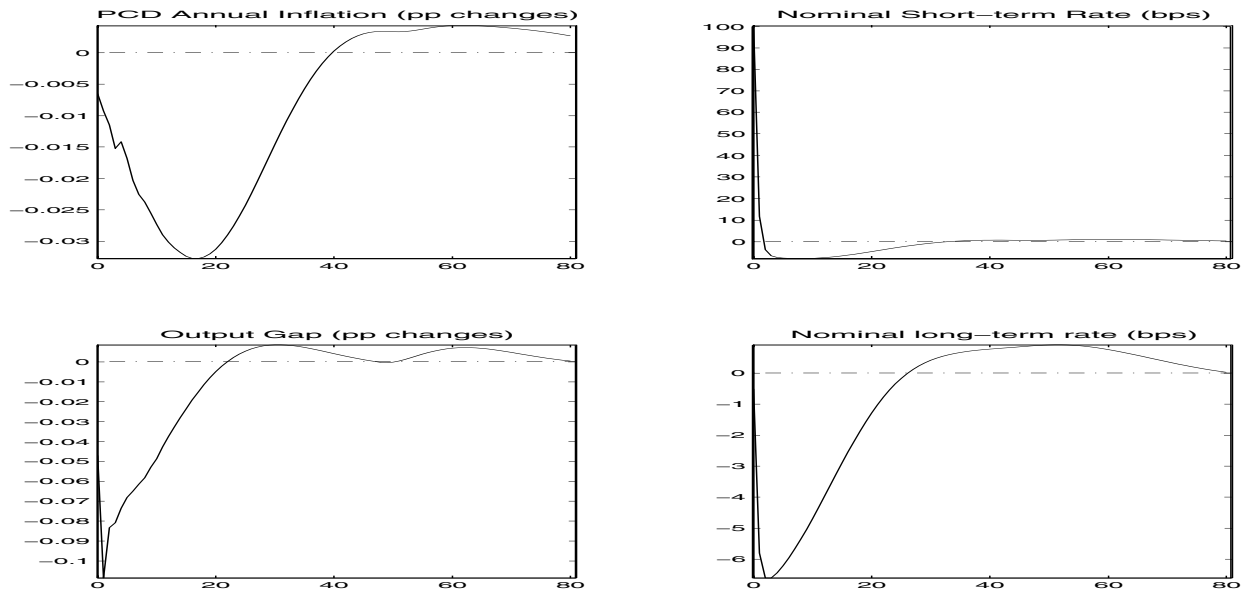

Figure 4: Impulse-responses to a 100bps temporary unanticipated monetary policy shock. The monetary policy rule is the Gerlach and Schnabel (2000) rule (6). All variables are measured in percentage points deviation from steady state.

year later. Smoother transition of short and long-term interest rates induces the output gap to show a stronger contraction of roughly $0.19 \%$ peaking six quarters after the shock and a secondary cycle reaching its peak of $0.08 \%$ above steady state almost nine years after the shock.

For the remaining two rules, the qualitative behavior is similar to rule (4) with the maximum amplitudes and the location of these depending on the degree of smoothing as in the two descriptions above. No effects can be singled out and attributed to the forward-lookingness involved. 
Summing up so far, the Area Wide Model is mostly backward-looking. It is characterized by a substantial amount of intrinsic persistence leading to a significant smoothing of interest rates even in the absence of an explicit smoothing term in the (generalized) Taylor rule. Long-term interest rates play a minor direct role in the model, so if the model were less persistent there would not be a substantial gain for the policymaker to control the entire yield curve. While too persistent policy may introduce substantial fluctuations, it is exactly the AWM's persistence that gives the policymaker a strong incentive for conducting forward-looking stabilization policy.

\subsection{The Taylor Principle - Determinacy Regions}

The Area Wide Model obeys one principle derived from the New-Keynesian paradigm; the "Taylor principle" states that nominal interest rates need to react more than one for one with inflation otherwise indeterminacy results, which is stressed, e.g., by Clarida et al. (1998) both from an empirical and theoretical perspective. While according to loss function (1) it is apparent that explosive equilibria can never be the result of optimal policy, we would argue that so would not be indeterminate equilibria as the policymaker cannot control the expectational errors. Figure 5 illustrates which parameter constellations induce indeterminacy, determinacy and explosiveness in the AWM. In the upper row, policy does not directly respond to the output gap, while the middle row shows a response of $\beta=0.4$ and the bottom row assumes a unit coefficient on the contemporaneous output gap. From left to right the forecast horizon for inflation is $\theta=0,4,8$ and 16 quarters, respectively. For each specification of forecast horizons and response to the output gap, the dark section shows the locus of $(\alpha, \rho)$ combinations for which the equilibrium is indeterminate, the white section indicates explosive solutions while the grey section indicates that such policy results in a determinate rational expectations equilibrium. Explosive solutions result when interest rate smoothing is too strong. For forecast horizons up to one year and no feedback from the output gap the explosive region in addition is increasing in the feedback to inflation (expectations). We find that the first difference rule, which Levin et al. (2003) recommend as a robust rule, $\rho=1, \alpha=0.4, \beta=0.4, \theta=4, \kappa=0$, is destabilizing within the AWM. As the forecast horizon in the policy rule increases, policy can be more inert - even superinertial rules lead to determinacy when the response to the inflation rate and the output gap are chosen adequately. 

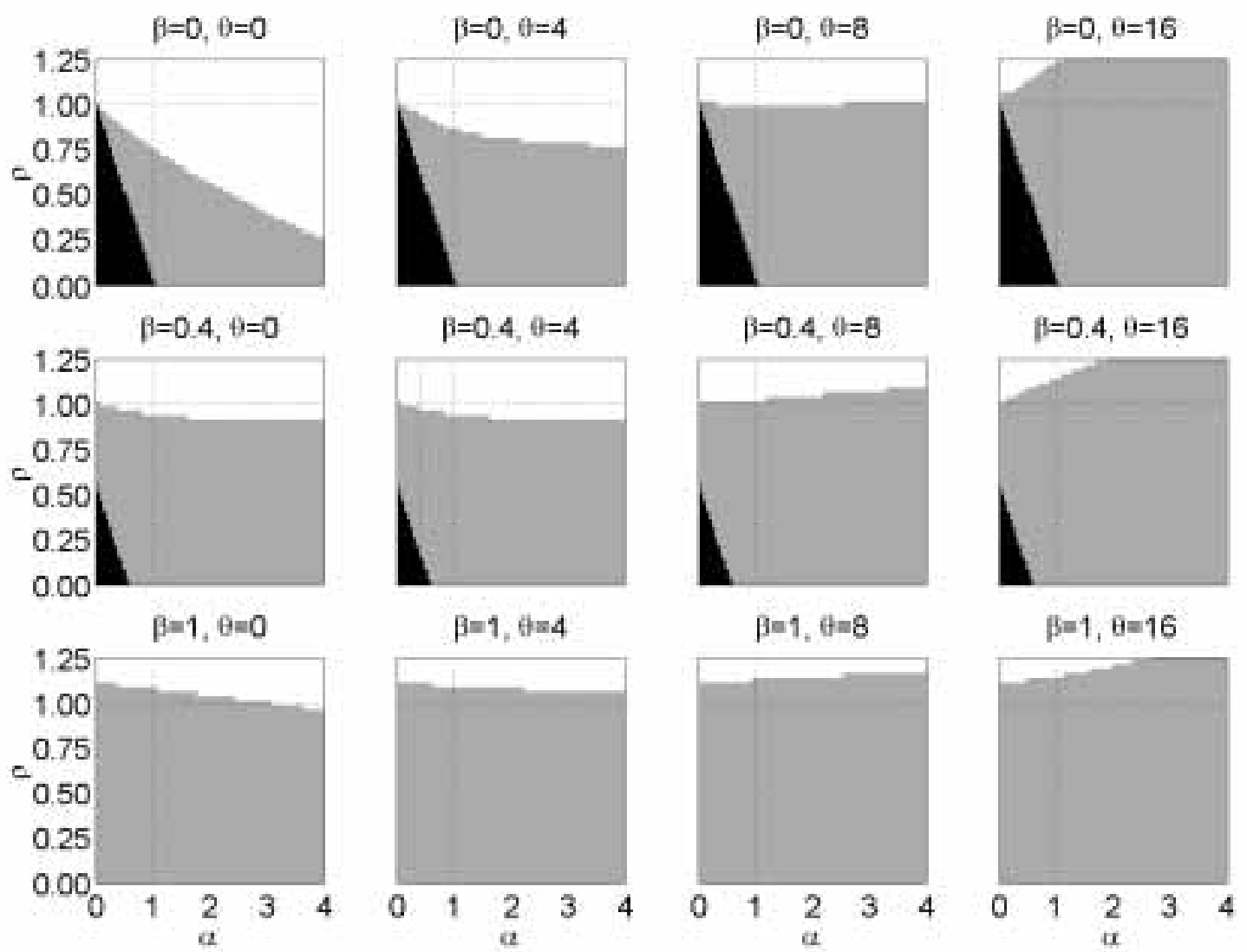

Figure 5: Determinacy regions. For varying responses to lagged interest rates, the contemporaneous output gap as well as to expectations of inflation at varying horizons, the panels display indeterminacy regions (dark), regions for explosive equilibria (white) and parameter constellations which result in a unique stationary rational expectations equilibrium (gray). The top row shows results for $\beta=0$, the middle row for $\beta=0.4$ and the bottom row for $\beta=1$. From left to right the columns report results for a response to contemporaneous annualized inflation, to 4 quarters ahead inflation expectations, to 8 and to 16 quarters ahead inflation expectations, respectively. The grids chosen are 0.025 for $\rho$ and 0.05 for $\alpha$. The horizontal and vertical dotted lines mark parameter values of $\rho=1$ and $\alpha=1$ respectively. For the case $\beta=0.4, \theta=4$, we draw an additional line at $\alpha=0.4$.

Indeterminacy occurs if the policy response to inflation is not strong enough. In line with the so called Taylor principle, for determinacy when there is no interest rate smoothing and no response to the output gap, the AWM requires a response of the nominal rate more than one for one with inflation. Even stricter, $\alpha$ needs to be larger than 1.05 in that case. Expanding the forecast horizon leaves the region of indeterminacy unaltered. The AWM appears to be relatively immune to indeterminacy problems associated with an increasing forecast horizon. This may be reconciled by the fact that the strong inflation (and output gap) persistence of the AWM both imply a close correspondence between the current inflation rate and its expected value several years into the future. 
Incorporating an explicit response to the current output gap shrinks both the indeterminacy and explosiveness regions for forecast horizons smaller than two years. The indeterminacy regions essentially vanish for $\beta=1$. Overall, including an output gap measure substantially improves the robustness of parameter choices for the policy rule with regard to indeterminacy and explosiveness. Results for more forward-looking models, as for example analyzed in Levin et al. (2003), show that a stronger smoothing of interest rates decreases the risk of ending up in an indeterminate region while not posing the risk of instability - a results we cannot subscribe to using the AWM. In contrast, too large a smoothing parameter may be inherently destabilizing at short forecast horizons. That indeterminacy regions decrease once the response to the output gap increases is in line with the more forward-looking modelling class.

Above, we have argued that the AWM presents a number of empirically reasonable deviations from the en vogue modelling paradigms and illustrated their implications for the model properties. We next turn to highlight how these features affect implications for optimal monetary policy.

\section{Optimal Policy}

Recalling our earlier discussion, the general form of the Taylor rule is:

$$
r_{t}=\rho r_{t-1}+\alpha E_{t} \pi_{t+\theta}^{q, a}+\beta E_{t} g a p_{t+\kappa}
$$

where inflation can be measured in annualized quarterly $(q)$ or year-on-year $(a)$ terms. In this section we minimize loss function (1) with respect to the reaction coefficients $\rho, \alpha$ and $\beta$ using three conventional informational assumptions regarding the horizons $\theta$ and $\kappa$ :

1. $\theta=\kappa=0$ contemporaneous information (Outcome-Based Rule)

2. $\theta=\kappa=-1$ lagged information (Outcome-Based Rule)

3. $\theta=4, \kappa=0$ one year forecast-based rule. $^{14}$

14 As regards $\kappa=0$, Batini and Haldane (1999) argue that monetary policy can effectively stabilize both inflation and output even through a rule with $\beta=0$, as long as the forecast horizon for inflation is 
Scenario 2 can be interpreted as reflecting a lack of (reliable) real time information. In scenario 3, we focus on a one-year forecast horizon, which has been the work-horse in the empirical literature. Throughout the paper, inflation rates are assumed to be quarterly in annual terms. ${ }^{15}$ We now turn to the issue of optimal monetary policy conduct evaluated by the quadratic loss function (1).

\subsection{Optimized Simple Rules vs. Optimal Discretionary Policy}

We choose the reaction coefficients $\rho, \alpha$ and $\beta$ over the rule scenarios 1 to 3 in Section 4 . Recalling our earlier discussion it is desirable to have a penalty on instrument variability. This may - as before - take the form of an explicit upper bound or a positive weight on interest rate variability in the loss function. In this section, we employ both measures. Firstly, when we obtain the optimal simple policy for a positive penalty, $\gamma$, we use values of $\gamma=0.01,0.1,0.5$ and 1.0. Secondly, as regards the precise value of the upper bound, we consider two solutions: a value consistent with the data, namely $\sigma_{\Delta r}=0.569$; and one which is sufficiently looser but still binding for any type of preference and any type of the generalized optimal simple Taylor rules (we take $\sigma_{\Delta r}=1.4$, which is roughly 2.5 times the empirical value). Table 2 summarizes the results for varying preferences for output gap stabilization if the contemporaneous Taylor Rule is used. Tables 3 and 4 report the results for the delayed informational assumption as well as for the forward-looking Taylor rule.

At extremes, we consider the case of strict inflation targeting, $\lambda=0$, and preferences geared towards output-gap targeting, $\lambda=3 .{ }^{16}$ Smoothing parameters $(\rho)$ for all informa-

positive. Their argument is that since output is a useful predictor of future inflation there is implicit "output-encompassing" in this case. A longer inflation forecast horizon brings inflation back to target more gradually dampening the amplitude of the real side on the outset of supply shocks. Varying $\theta$ alone then varies the degree of encompassing. Levin et al. (2003) have an empirical counter-example where setting $\beta=0$ considerably deteriorates stabilization performance.

${ }^{15}$ Levin et al. (1999) argue that high frequency noise may lead the policymaker not to base his interest rate decisions on quarterly inflation information but rather on annual inflation in order to average this noise out. We examined this argument for a subset of simple rules. For contemporaneous rules there is a slight advantage of using annual information in the feedback rules. For forecast-based rules $(\theta=4)$ this advantage vanishes as one would have expected since in forming expectations high-frequency noise does not play any role (the model is linear and shocks are additive white noise). In addition, expected quarterly inflation a year ahead is better controllable than expected annual inflation a year ahead, as the latter includes the intermediate quarterly inflation rates which are harder to influence due to policy lags. Qualitative results, however, did not seem to depend much on this choice.

16 Note that for the direct punishment cases, $\gamma>0$, the relative weight on smoothing the interest rate 
Table 2: Optimized Policy Under Contemporaneous Rule ${ }^{a}$

\begin{tabular}{|c|c|c|c|c|c|c|c|c|c|}
\hline $\bar{\lambda}$ & $\gamma$ & $\rho$ & $\alpha$ & $\bar{\beta}$ & $\sigma_{\text {gap }}$ & $\overline{\sigma_{\pi}}$ & $\sigma_{\Delta r}$ & $\overline{\sigma_{r}}$ & $\overline{L o s s}$ \\
\hline \multicolumn{10}{|c|}{$\bar{\sigma}_{\Delta r}=0.569$} \\
\hline 0.00 & 0.00 & 0.77 & 0.24 & 0.33 & 2.00 & 1.19 & 0.57 & 2.69 & 1.42 \\
\hline 0.10 & 0.00 & 0.74 & 0.23 & 0.37 & 1.95 & 1.19 & 0.57 & 2.59 & 1.80 \\
\hline 0.33 & 0.00 & 0.70 & 0.21 & 0.41 & 1.91 & 1.20 & 0.57 & 2.50 & 2.67 \\
\hline 1.00 & 0.00 & 0.66 & 0.18 & 0.45 & 1.90 & 1.22 & 0.57 & 2.44 & 5.08 \\
\hline 3.00 & 0.00 & 0.64 & 0.17 & 0.47 & 1.89 & 1.23 & 0.57 & 2.41 & 12.26 \\
\hline \multicolumn{10}{|c|}{$\bar{\sigma}_{\Delta r}=1.4$} \\
\hline 0.00 & 0.00 & 0.56 & 0.86 & 1.13 & 1.49 & 1.07 & 1.40 & 3.97 & 1.15 \\
\hline 0.10 & 0.00 & 0.50 & 0.61 & 1.45 & 1.36 & 1.08 & 1.40 & 3.89 & 1.35 \\
\hline 0.33 & 0.00 & 0.47 & 0.39 & 1.63 & 1.32 & 1.09 & 1.40 & 3.85 & 1.76 \\
\hline 1.00 & 0.00 & 0.46 & 0.25 & 1.69 & 1.30 & 1.10 & 1.40 & 3.83 & 2.90 \\
\hline 3.00 & 0.00 & 0.45 & 0.18 & 1.72 & 1.30 & 1.10 & 1.40 & 3.82 & 6.29 \\
\hline \multicolumn{10}{|c|}{$\overline{\bar{\sigma}}_{\Delta r}$ free } \\
\hline 0.00 & 0.01 & 0.43 & 1.77 & 2.00 & 1.27 & 1.04 & 2.29 & 4.85 & 1.13 \\
\hline 0.10 & 0.01 & 0.37 & 1.68 & 3.83 & 0.93 & 1.04 & 2.91 & 5.52 & 1.25 \\
\hline 0.33 & 0.01 & 0.41 & 1.42 & 6.32 & 0.69 & 1.05 & 3.78 & 6.31 & 1.40 \\
\hline 1.00 & 0.01 & 0.48 & 1.07 & 9.74 & 0.52 & 1.05 & 4.93 & 7.00 & 1.63 \\
\hline 3.00 & 0.01 & 0.53 & 0.74 & 14.71 & 0.41 & 1.06 & 6.42 & 7.62 & 2.04 \\
\hline \multicolumn{10}{|c|}{$\bar{\sigma}_{\Delta r}$ free } \\
\hline 0.00 & 0.10 & 0.64 & 0.58 & 0.80 & 1.63 & 1.10 & 1.07 & 3.55 & 1.32 \\
\hline 0.10 & 0.10 & 0.51 & 0.57 & 1.35 & 1.40 & 1.08 & 1.33 & 3.80 & 1.54 \\
\hline 0.33 & 0.10 & 0.43 & 0.49 & 2.25 & 1.16 & 1.07 & 1.78 & 4.38 & 1.91 \\
\hline 1.00 & 0.10 & 0.44 & 0.39 & 3.65 & 0.91 & 1.07 & 2.51 & 5.27 & 2.59 \\
\hline 3.00 & 0.10 & 0.49 & 0.29 & 5.80 & 0.69 & 1.07 & 3.52 & 6.18 & 3.80 \\
\hline \multicolumn{10}{|c|}{$\bar{\sigma}_{\Delta r}$ free } \\
\hline 0.00 & 0.50 & 0.75 & 0.28 & 0.39 & 1.93 & 1.17 & 0.64 & 2.83 & 1.57 \\
\hline 0.10 & 0.50 & 0.67 & 0.31 & 0.57 & 1.77 & 1.14 & 0.76 & 2.92 & 1.91 \\
\hline 0.33 & 0.50 & 0.54 & 0.30 & 0.97 & 1.56 & 1.12 & 0.98 & 3.20 & 2.55 \\
\hline 1.00 & 0.50 & 0.46 & 0.25 & 1.68 & 1.31 & 1.10 & 1.39 & 3.81 & 3.88 \\
\hline 3.00 & 0.50 & 0.45 & 0.20 & 2.84 & 1.03 & 1.08 & 2.08 & 4.77 & 6.52 \\
\hline \multicolumn{10}{|c|}{$\bar{\sigma}_{\Delta r}$ free } \\
\hline 0.00 & 1.00 & 0.79 & 0.21 & 0.29 & 2.05 & 1.21 & 0.51 & 2.57 & 1.73 \\
\hline 0.10 & 1.00 & 0.73 & 0.24 & 0.38 & 1.93 & 1.19 & 0.59 & 2.62 & 2.13 \\
\hline 0.33 & 1.00 & 0.62 & 0.25 & 0.64 & 1.74 & 1.16 & 0.75 & 2.81 & 2.90 \\
\hline 1.00 & 1.00 & 0.49 & 0.22 & 1.16 & 1.49 & 1.12 & 1.06 & 3.28 & 4.60 \\
\hline 3.00 & 1.00 & 0.45 & 0.18 & 2.03 & 1.21 & 1.09 & 1.60 & 4.12 & 8.15 \\
\hline
\end{tabular}

${ }^{a}$ Optimal outcome-based policy, $\theta=\kappa=0$, under full commitment to rule (2).

is decreasing in the level of $\lambda$. In other words, only the strict upper bound cases provide genuine comparisons all else equal. 
Table 3: Optimized Policy Under Lagged Rule ${ }^{a}$

\begin{tabular}{|c|c|c|c|c|c|c|c|c|c|}
\hline$\lambda$ & $\gamma$ & $\rho$ & $\alpha$ & $\beta$ & $\sigma_{\text {gap }}$ & $\sigma_{\pi}$ & $\sigma_{\Delta r}$ & $\overline{\sigma_{r}}$ & Loss \\
\hline \multicolumn{10}{|c|}{$\bar{\sigma}_{\Delta r}=0.569$} \\
\hline 0.00 & 0.00 & 0.76 & 0.23 & 0.32 & 2.16 & 1.24 & 0.57 & 2.72 & 1.52 \\
\hline 0.10 & 0.00 & 0.72 & 0.23 & 0.36 & 2.12 & 1.24 & 0.57 & 2.61 & 1.98 \\
\hline 0.33 & 0.00 & 0.67 & 0.22 & 0.39 & 2.08 & 1.25 & 0.57 & 2.51 & 3.01 \\
\hline 1.00 & 0.00 & 0.63 & 0.20 & 0.43 & 2.07 & 1.26 & 0.57 & 2.44 & 5.88 \\
\hline 3.00 & 0.00 & 0.60 & 0.19 & 0.45 & 2.06 & 1.28 & 0.57 & 2.40 & 14.40 \\
\hline \multicolumn{10}{|c|}{$\bar{\sigma}_{\Delta r}=1.4$} \\
\hline 0.00 & 0.00 & 0.54 & 0.71 & 1.09 & 1.72 & 1.11 & 1.40 & 4.11 & 1.22 \\
\hline 0.10 & 0.00 & 0.44 & 0.53 & 1.34 & 1.62 & 1.11 & 1.40 & 3.91 & 1.50 \\
\hline 0.33 & 0.00 & 0.38 & 0.37 & 1.50 & 1.58 & 1.12 & 1.40 & 3.80 & 2.09 \\
\hline 1.00 & 0.00 & 0.35 & 0.25 & 1.58 & 1.57 & 1.13 & 1.40 & 3.75 & 3.75 \\
\hline 3.00 & 0.00 & 0.34 & 0.19 & 1.61 & 1.57 & 1.14 & 1.40 & 3.72 & 8.68 \\
\hline \multicolumn{10}{|c|}{$\bar{\sigma}_{\Delta r}$ free } \\
\hline 0.00 & 0.01 & 0.38 & 1.38 & 1.99 & 1.50 & 1.07 & 2.32 & 5.10 & 1.20 \\
\hline 0.10 & 0.01 & 0.24 & 1.19 & 3.43 & 1.24 & 1.07 & 3.02 & 5.62 & 1.38 \\
\hline 0.33 & 0.01 & 0.20 & 0.89 & 4.96 & 1.07 & 1.07 & 4.01 & 6.36 & 1.69 \\
\hline 1.00 & 0.01 & 0.14 & 0.64 & 6.67 & 0.96 & 1.07 & 5.35 & 7.11 & 2.35 \\
\hline 3.00 & 0.01 & 0.04 & 0.53 & 8.58 & 0.89 & 1.07 & 7.05 & 7.84 & 4.03 \\
\hline \multicolumn{10}{|c|}{$\bar{\sigma}_{\Delta r}$ free } \\
\hline 0.00 & 0.10 & 0.60 & 0.53 & 0.82 & 1.83 & 1.13 & 1.12 & 3.72 & 1.40 \\
\hline 0.10 & 0.10 & 0.44 & 0.52 & 1.31 & 1.63 & 1.11 & 1.37 & 3.87 & 1.69 \\
\hline 0.33 & 0.10 & 0.32 & 0.43 & 2.07 & 1.45 & 1.10 & 1.81 & 4.34 & 2.24 \\
\hline 1.00 & 0.10 & 0.27 & 0.31 & 3.16 & 1.25 & 1.09 & 2.58 & 5.18 & 3.42 \\
\hline 3.00 & 0.10 & 0.24 & 0.22 & 4.60 & 1.08 & 1.08 & 3.70 & 6.13 & 6.06 \\
\hline \multicolumn{10}{|c|}{$\bar{\sigma}_{\Delta r}$ free } \\
\hline 0.00 & 0.50 & 0.72 & 0.28 & 0.40 & 2.09 & 1.20 & 0.67 & 2.92 & 1.67 \\
\hline 0.10 & 0.50 & 0.63 & 0.31 & 0.56 & 1.95 & 1.18 & 0.77 & 2.96 & 2.08 \\
\hline 0.33 & 0.50 & 0.48 & 0.31 & 0.93 & 1.78 & 1.16 & 0.98 & 3.19 & 2.88 \\
\hline 1.00 & 0.50 & 0.35 & 0.25 & 1.56 & 1.58 & 1.13 & 1.39 & 3.72 & 4.73 \\
\hline 3.00 & 0.50 & 0.29 & 0.18 & 2.53 & 1.36 & 1.10 & 2.09 & 4.63 & 8.92 \\
\hline \multicolumn{10}{|c|}{$\bar{\sigma}_{\Delta r}$ free } \\
\hline 0.00 & 1.00 & 0.77 & 0.21 & 0.29 & 2.19 & 1.25 & 0.53 & 2.64 & 1.85 \\
\hline 0.10 & 1.00 & 0.70 & 0.24 & 0.38 & 2.09 & 1.23 & 0.60 & 2.66 & 2.31 \\
\hline 0.33 & 1.00 & 0.58 & 0.26 & 0.61 & 1.93 & 1.20 & 0.75 & 2.81 & 3.24 \\
\hline 1.00 & 1.00 & 0.42 & 0.24 & 1.09 & 1.73 & 1.16 & 1.05 & 3.22 & 5.44 \\
\hline 3.00 & 1.00 & 0.32 & 0.19 & 1.86 & 1.50 & 1.12 & 1.58 & 3.98 & 10.55 \\
\hline
\end{tabular}

$\bar{a}$ Optimal lagged information policy, $\theta=\kappa=-1$, under full commitment to rule (2). 
Table 4: Optimized Policy Under Forecast-Based Rule ${ }^{a}$

\begin{tabular}{|c|c|c|c|c|c|c|c|c|c|}
\hline $\bar{\lambda}$ & $\gamma$ & $\rho$ & $\alpha$ & $\bar{\beta}$ & $\sigma_{\text {gap }}$ & $\overline{\sigma_{\pi}}$ & $\sigma_{\Delta r}$ & $\overline{\sigma_{r}}$ & $\overline{L o s s}$ \\
\hline \multicolumn{10}{|c|}{$\bar{\sigma}_{\Delta r}=0.569$} \\
\hline 0.00 & 0.00 & 0.67 & 1.12 & 0.21 & 1.83 & 1.09 & 0.57 & 2.56 & 1.18 \\
\hline 0.10 & 0.00 & 0.64 & 1.05 & 0.26 & 1.78 & 1.09 & 0.57 & 2.46 & 1.50 \\
\hline 0.33 & 0.00 & 0.61 & 0.97 & 0.31 & 1.76 & 1.10 & 0.57 & 2.39 & 2.23 \\
\hline 1.00 & 0.00 & 0.59 & 0.89 & 0.36 & 1.75 & 1.11 & 0.57 & 2.34 & 4.27 \\
\hline 3.00 & 0.00 & 0.57 & 0.84 & 0.38 & 1.74 & 1.11 & 0.57 & 2.31 & 10.35 \\
\hline \multicolumn{10}{|c|}{$\bar{\sigma}_{\Delta r}=1.4$} \\
\hline 0.00 & 0.00 & 0.52 & 3.84 & 0.46 & 1.49 & 0.99 & 1.40 & 3.84 & 0.97 \\
\hline 0.10 & 0.00 & 0.50 & 3.04 & 0.85 & 1.31 & 1.00 & 1.40 & 3.75 & 1.16 \\
\hline 0.33 & 0.00 & 0.49 & 2.35 & 1.12 & 1.25 & 1.01 & 1.40 & 3.76 & 1.54 \\
\hline 1.00 & 0.00 & 0.48 & 1.88 & 1.28 & 1.23 & 1.02 & 1.40 & 3.76 & 2.56 \\
\hline 3.00 & 0.00 & 0.47 & 1.65 & 1.35 & 1.23 & 1.03 & 1.40 & 3.76 & 5.58 \\
\hline \multicolumn{10}{|c|}{$\overline{\bar{\sigma}}_{\Delta r}$ free } \\
\hline 0.00 & 0.01 & 0.45 & 7.65 & 0.57 & 1.46 & 0.95 & 2.36 & 5.11 & 0.96 \\
\hline 0.10 & 0.01 & 0.39 & 6.89 & 1.96 & 1.02 & 0.96 & 2.67 & 5.12 & 1.11 \\
\hline 0.33 & 0.01 & 0.39 & 6.35 & 4.20 & 0.74 & 0.99 & 3.46 & 5.85 & 1.28 \\
\hline 1.00 & 0.01 & 0.42 & 5.39 & 7.74 & 0.54 & 1.01 & 4.61 & 6.64 & 1.53 \\
\hline 3.00 & 0.01 & 0.46 & 4.37 & 12.94 & 0.41 & 1.03 & 6.15 & 7.38 & 1.96 \\
\hline \multicolumn{10}{|c|}{$\bar{\sigma}_{\Delta r}$ free } \\
\hline 0.00 & 0.10 & 0.58 & 2.38 & 0.37 & 1.60 & 1.02 & 0.99 & 3.26 & 1.13 \\
\hline 0.10 & 0.10 & 0.52 & 2.56 & 0.71 & 1.39 & 1.01 & 1.22 & 3.50 & 1.35 \\
\hline 0.33 & 0.10 & 0.47 & 2.79 & 1.41 & 1.15 & 1.00 & 1.65 & 4.11 & 1.72 \\
\hline 1.00 & 0.10 & 0.45 & 2.95 & 2.71 & 0.90 & 1.01 & 2.37 & 5.01 & 2.39 \\
\hline 3.00 & 0.10 & 0.46 & 2.96 & 4.84 & 0.68 & 1.02 & 3.35 & 5.94 & 3.56 \\
\hline \multicolumn{10}{|c|}{$\bar{\sigma}_{\Delta r}$ free } \\
\hline 0.00 & 0.50 & 0.66 & 1.15 & 0.22 & 1.82 & 1.08 & 0.58 & 2.58 & 1.34 \\
\hline 0.10 & 0.50 & 0.61 & 1.27 & 0.33 & 1.70 & 1.06 & 0.67 & 2.64 & 1.65 \\
\hline 0.33 & 0.50 & 0.54 & 1.47 & 0.59 & 1.53 & 1.04 & 0.88 & 2.93 & 2.25 \\
\hline 1.00 & 0.50 & 0.48 & 1.76 & 1.16 & 1.28 & 1.03 & 1.30 & 3.61 & 3.53 \\
\hline 3.00 & 0.50 & 0.46 & 2.12 & 2.19 & 1.00 & 1.02 & 1.98 & 4.58 & 6.03 \\
\hline \multicolumn{10}{|c|}{$\bar{\sigma}_{\Delta r}$ free } \\
\hline 0.00 & 1.00 & 0.70 & 0.86 & 0.16 & 1.91 & 1.12 & 0.46 & 2.35 & 1.47 \\
\hline 0.10 & 1.00 & 0.65 & 0.96 & 0.23 & 1.82 & 1.10 & 0.52 & 2.38 & 1.82 \\
\hline 0.33 & 1.00 & 0.59 & 1.11 & 0.39 & 1.68 & 1.08 & 0.66 & 2.55 & 2.53 \\
\hline 1.00 & 1.00 & 0.51 & 1.37 & 0.77 & 1.46 & 1.05 & 0.97 & 3.05 & 4.15 \\
\hline 3.00 & 1.00 & 0.47 & 1.75 & 1.50 & 1.18 & 1.03 & 1.51 & 3.94 & 7.50 \\
\hline
\end{tabular}

a Optimal forecast-based information policy, $\theta=4, \kappa=0$, under full commitment to rule (2). 
tional assumptions are rather small, ranging between 0.04 and 0.79 across preferences and policy rules. These values are thus far from unity even for a strict inflation target - in contrast to the results of Levin et al. $(1999,2003)$ for Federal Reserve Board models. The features of the AWM outlined in section 3 provide some explanations: first, abstracting from the intrinsic persistence in inflation and the output gap, long-term interest rates and expectations do not play a dominant role in the AWM. The commitment value of persistent changes in the real rates is not substantial enough so as to merit strong additional smoothing. Second, as highlighted in section 3 even a rule with modest smoothing can imply substantial smoothing of the policy instrument in the reduced form of the model due to the model's intrinsic persistence. In light of the less pronounced smoothing found here, it is not surprising that the integral control or first-difference rule purported in Levin et al. (2003) to be robust to model uncertainty, does not even succeed in stabilizing the economy but results in an explosive equilibrium. These results for the AWM square with the evidence of Batini and Nelson (2001), Côté et al. (2002) as well as Levin and Williams (2003): in the more backward-looking and persistent models smoothing is less pronounced and excessive smoothing may severely deteriorate stabilization.

Another notable feature is that within each class of policies, the possible percentage reductions in output variability and inflation variability both are small, being close to $5 \%$ and $3 \%$, respectively, for the case of the empirical upper bound. For the contemporaneous outcome-based rule, for example, output variability is reduced by $5.3 \%$ when moving from to $\lambda=0$ to $\lambda=3$, while inflation volatility is only reduced by $3.1 \%$ going in the opposite direction. However, this does not mean that there is barely any menu choice for the policymaker: comparing the economic stability under the optimal rules to the performance under the given rules in Table 1 highlights that there is a substantial stabilization gain arising from optimal monetary policy.

Allowing for more flexibility in interest rates shows that the AWM implies that the largest gain from stabilization can be imparted on the output gap while the reduction in inflation variability is comparatively mild. For instance, for the contemporaneous Taylor rule with an upper bound on interest rate change volatility of 1.4, output variability is reduced by $15 \%$ while inflation variability only increases by roughly $3 \%$. On the one hand, there may be a strong "exogenous" component in the price dynamics, on the other, given the long cycles in output illustrated in figures 1 through 4 , curbing real side variability 
may contribute substantially towards stabilizing inflation.Imposing no strict upper bound on interest change variability, but rather implementing a penalty term $\gamma>0$ in the loss function illustrates that there is a strong trade-off between stabilizing the output gap and stabilizing the interest rate, while again the trade-off for inflation stabilization is rather mild. ${ }^{17}$ As argued in section 2 , the implications of optimal discretionary policy provide a natural benchmark - reported in Table 5 .

Figure 6 highlights for the case of the empirical upper bound that there is hardly any "menu choice" within each set of policies for the policymaker once conducting optimal policy. However, as we plot the efficiency frontiers for the policies considered herein, there are substantial stabilization differences across classes of policies. The respective northwesterly part of the frontiers pertains to an inflation targeting central bank while the southeast refers to almost pure output gap targeting. Three observations are apparent: first, using current information is substantially preferred to using lagged information. Second, for the arbitrary choice of a one-year forecast horizon for inflation, forecast-based policy strictly dominates outcome-based policy. Third, there is substantial value added of incorporating all information into the model as highlighted by the stabilization performance of optimal discretionary policy. For empirical comparison, we also show two rules recently estimated for the Euro area: Gerdesmeier and Roffia's (2003) rule with contemporaneous inflation and Gerlach and Schnabel's (2000) rule with a forecast based inflation term. While in the AWM these imply slightly larger interest rate variability than observed in the data, they can nevertheless serve as a rough benchmark for the simple optimal rules. In particular, the Gerdesmeier and Roffia rule puts insufficient weight on the output gap and hence does considerably worse than the optimal contemporaneous outcome-based rule. In contrast, by visual inspection, the rule estimated by Gerlach and Schnabel, although incorporating insufficient smoothing, comes closer to the optimal frontier for forecast-based policy.

Simple rules may be argued to be easy to monitor so the central bank may be able to commit to them credibly. However, being contingent only on a small subset of states,

17 This may even look inverted, i.e. as if inflation variability would be decreasing in the weight on output stability. However, increasing $\lambda$ alters both the inflation-output gap stabilization trade-off and the trade-off between stabilizing either of these and smoothing the interest rate. Namely the latter is relatively less costly when $\lambda$ is increased, rendering decreases of inflation variability if $\lambda$ increases plausible. 
Table 5: Results for Optimal Discretionary Policy ${ }^{a}$

\begin{tabular}{|c|c|c|c|c|c|c|}
\hline $\bar{\lambda}$ & $\gamma$ & $\sigma_{g a p}$ & $\sigma_{\pi}$ & $\sigma_{\Delta r}$ & $\sigma_{r}$ & Loss \\
\hline \multicolumn{7}{|c|}{$\bar{\sigma}_{\Delta r}=0.569$} \\
\hline 0.00 & 0.00 & 1.66 & 1.01 & 0.57 & 2.39 & 1.01 \\
\hline 0.10 & 0.00 & 1.55 & 1.01 & 0.57 & 2.28 & 1.27 \\
\hline 0.33 & 0.00 & 1.49 & 1.03 & 0.57 & 2.22 & 1.80 \\
\hline 1.00 & 0.00 & 1.47 & 1.05 & 0.57 & 2.18 & 3.27 \\
\hline 3.00 & 0.00 & 1.47 & 1.06 & 0.57 & 2.16 & 7.61 \\
\hline \multicolumn{7}{|c|}{$\overline{\bar{\sigma}_{\Delta r}=1.4}$} \\
\hline 0.00 & 0.00 & 1.59 & 0.95 & 1.40 & 3.81 & 0.91 \\
\hline 0.10 & 0.00 & 1.21 & 0.97 & 1.40 & 3.59 & 1.08 \\
\hline 0.33 & 0.00 & 1.10 & 0.99 & 1.40 & 3.60 & 1.38 \\
\hline 1.00 & 0.00 & 1.07 & 1.01 & 1.40 & 3.61 & 2.15 \\
\hline 3.00 & 0.00 & 1.06 & 1.02 & 1.40 & 3.61 & 4.42 \\
\hline \multicolumn{7}{|c|}{$\bar{\sigma}_{\Delta r}$ free } \\
\hline 0.00 & 0.01 & 1.64 & 0.94 & 2.11 & 4.84 & 0.92 \\
\hline 0.10 & 0.01 & 1.04 & 0.95 & 2.40 & 4.74 & 1.07 \\
\hline 0.33 & 0.01 & 0.75 & 0.98 & 3.17 & 5.50 & 1.24 \\
\hline 1.00 & 0.01 & 0.54 & 1.01 & 4.32 & 6.34 & 1.49 \\
\hline 3.00 & 0.01 & 0.41 & 1.03 & 5.86 & 7.14 & 1.91 \\
\hline \multicolumn{7}{|c|}{$\bar{\sigma}_{\Delta r}$ free } \\
\hline 0.00 & 0.10 & 1.61 & 0.98 & 0.82 & 2.87 & 1.03 \\
\hline 0.10 & 0.10 & 1.34 & 0.98 & 1.00 & 3.02 & 1.24 \\
\hline 0.33 & 0.10 & 1.10 & 0.99 & 1.41 & 3.62 & 1.58 \\
\hline 1.00 & 0.10 & 0.86 & 1.00 & 2.10 & 4.54 & 2.19 \\
\hline 3.00 & 0.10 & 0.66 & 1.02 & 3.06 & 5.53 & 3.30 \\
\hline \multicolumn{7}{|c|}{$\bar{\sigma}_{\Delta r}$ free } \\
\hline 0.00 & 0.50 & 1.70 & 1.02 & 0.47 & 2.19 & 1.16 \\
\hline 0.10 & 0.50 & 1.57 & 1.02 & 0.54 & 2.22 & 1.43 \\
\hline 0.33 & 0.50 & 1.40 & 1.01 & 0.71 & 2.48 & 1.94 \\
\hline 1.00 & 0.50 & 1.19 & 1.01 & 1.09 & 3.12 & 3.04 \\
\hline 3.00 & 0.50 & 0.96 & 1.02 & 1.72 & 4.07 & 5.26 \\
\hline \multicolumn{7}{|c|}{$\bar{\sigma}_{\Delta r}$ free } \\
\hline 0.00 & 1.00 & 1.75 & 1.05 & 0.38 & 1.98 & 1.24 \\
\hline 0.10 & 1.00 & 1.65 & 1.04 & 0.42 & 1.98 & 1.53 \\
\hline 0.33 & 1.00 & 1.52 & 1.03 & 0.53 & 2.14 & 2.12 \\
\hline 1.00 & 1.00 & 1.33 & 1.03 & 0.79 & 2.60 & 3.46 \\
\hline 3.00 & 1.00 & 1.10 & 1.02 & 1.28 & 3.43 & 6.35 \\
\hline
\end{tabular}

${ }^{a}$ Optimal discretionary policy under full information. See Appendix

E for details for the upper bound cases. 


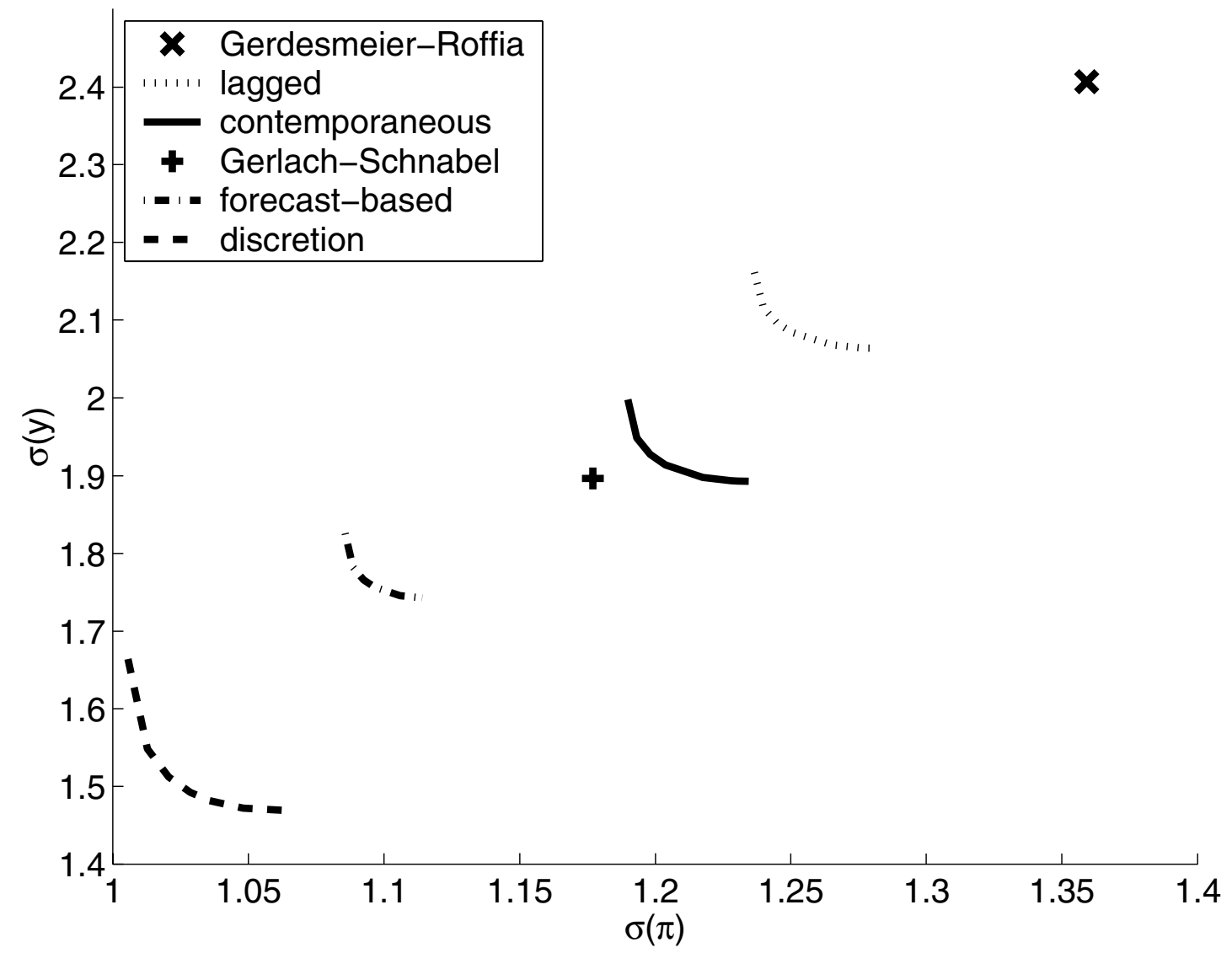

Figure 6: Optimal Policy Frontiers. Shown is the locus of combinations of standard deviations of the output gap (vertical axis) and standard deviations of inflation (horizontal axis) for the optimized simple lagged (dotted) and contemporaneous (solid) outcome-based rules as well as for the forecast-based (dash-dotted) rule with a horizons $\theta=4, \kappa=0$ for the empirical upper bound on interest rate change variability. In addition, we plot the locus implied by rules (4) and (6). The frontier implied by optimal policy under discretion is shown as a dashed line.

these are neither fully optimal nor necessarily time-consistent in the absence of some commitment technology. While it is commonly found that the commitment value provided by credible Taylor rules outweighs the loss incurred by conditioning policy only on a subset of information, this ranking depends on model characteristics. Most notably the greater is the information content of other states in the economy (besides the output gap, interest rates and inflation) and the less important is the expectations channel (in the extreme, in a backward-looking model, there is no distinction between Discretion and Commitment), the more valuable will be optimal time-consistent policy as opposed to sub-optimal (simple optimal) policy under commitment. We provide the optimal discretionary solution as a natural benchmark in the spirit of inflation targeting as promoted by Svensson (1999a). 
For the AWM, due to its highly persistent and rich structure, the information gain by far outweighs the credibility loss when we compare the simple optimal frontiers to the optimal discretionary frontier. Consequently, one might conjecture, that the information encompassing property of the forecast-based rules explains most of the gains relative to contemporaneous rules. In contrast to other authors, such as Levin et al. (2003), we find the gains from implementing forecast-based policy to be marked. Table 6 presents

Table 6: Relative Losses ${ }^{a}$

\begin{tabular}{||c||c||c|c|c||}
\hline \hline Rule & $\%$ Loss & $\rho$ & $\alpha$ & $\beta$ \\
\hline \hline \multicolumn{5}{||c||}{ Strict inflation targeter, $\lambda=0$} \\
\hline Contemporaneous & - & 0.77 & 0.24 & 0.33 \\
\hline Lagged & 7.98 & 0.76 & 0.23 & 0.32 \\
\hline Forecast-Based & -16.76 & 0.67 & 1.12 & 0.21 \\
\hline Discretion & -28.58 & - & - & - \\
\hline \hline \multicolumn{5}{||c||}{ Flexible inflation targeter, $\lambda=1$} \\
\hline Contemporaneous & - & 0.66 & 0.18 & 0.45 \\
\hline Lagged & 15.62 & 0.63 & 0.20 & 0.43 \\
\hline Forecast-Based & -15.99 & 0.59 & 0.89 & 0.36 \\
\hline Discretion & -35.74 & - & - & - \\
\hline \hline
\end{tabular}

$a \%$ Loss is the percentage increase in loss relative to the loss under the respective optimized simple contemporaneous rule for $\lambda=0$ and $\lambda=1$, respectively. The Table refers to the results with an empirical upper bound of $\bar{\sigma}_{\Delta r}=0.569$ on interest rate change variability.

numerical values for the relative losses and illustrates that the gain from implementing forecast-based policy is roughly $17 \%$ relative to implementing an optimized Taylor rule feeding back on contemporaneous inflation. Discretionary policy using all states of the model by far outperforms the simple rules under commitment (a reduction in losses of roughly $29 \%$ relative to contemporaneous Taylor rules). This highlights that there is a substantial amount of information in the model for which inflation and the output gap are not sufficient statistics. While the exchange rate as demonstrated in (McAdam and Morgan, 2003), plays a key role in the model, and thereby its expectations, the 
expectations mechanism is not strong enough to outweigh the loss of information due to not feeding back on all states. ${ }^{18}$

As Table 2 highlights, for outcome-based Taylor rules, the weight $\beta$ on the output gap is always larger than the weight on the inflation term even for a strictly inflation targeting central bank. While output is not a target variable in that case, it will in light of the impulse response evidence of section 3 be a good indicator variable for expected future inflation, which given policy lags ultimately is what the central bank is able to influence. Consequently, the weight on the output gap term decreases substantially relative to the inflation weight, when the policymaker is allowed to directly react to expected inflation.

Our results so far highlight first, that simple rules can do much worse than optimal discretionary policy. After all, in a more backward-looking but persistent framework, where most of the variables are pre-determined, the central bank needs to take most of those into account for optimal policy conduct. Second, in line with these results, the forward-looking Taylor rules provide a remarkable improvement above outcome-based rules. We attribute these results to the information-encompassing provided by the rational expectations in the rule. Despite the apparent structural simplicity of the simple rules, for practical policy implementation several caveats seem to be in order. The outcomebased rules are easily implemented only if one abstracts from the uncertainty surrounding (real-time) output gap estimates. In addition, expectations in the policy rule and in the model are taken to be model consistent rational expectations. Among other things, this amounts to the assumption that private sector forecasts are derived using the central bank's structural (Area-Wide) model - which may be violated in the face of model uncertainty. While this assumption also has a bearing on the optimal parameter values of the rules, its importance may be most obvious for forecast based rules. Consequently, to guard against private sector expectations not being in line with central bank forecasts, one may be tempted to resort to survey forecasts instead of applying model-consistent forecasts. Bernanke and Woodford (1997), however, illustrate that things are not that straightforward. In particular, directly targeting survey private sector expectations would

${ }^{18}$ From a theoretical angle, Svensson (2000) has argued that foreign variables should also appear as feedback parameters in open economy Taylor rules. From an empirical perspective Clarida et al. (1998) illustrate that real exchange rates and foreign funds rates could be significant ingredients to the optimized simple rule, although they have small quantitative effects. Gerdesmeier and Roffia (2003) find that the real effective exchange rate is just marginally insignificant, while the significance of nominal exchange rates and the federal funds rate is refuted more soundly. Upon a first exploration we did not find a few contemporaneous variables capturing the bulk of information in the model. We stick to above set of variables in order to keep our results comparable to the closed economy literature. 
likely nullify the information content of these measures and thereby lead the procedure ad absurdum leaving the equilibrium open to indeterminacy. Ultimately then, monetary authorities must rely on an explicit structural model of the economy to guide their policy decisions, as we do here, and cannot point at the simplicity of these rules.

In section 5, we derive optimal forecast horizons for forecast-based policy rules. We will illustrate that there is significant gain from an optimal choice of the horizon both for inflation as well as for the output gap forecast. Intuitively speaking, this feature arises due to the exploitation of all model states (although in a restricted manner). Before turning to the optimal horizons we briefly highlight the role of commitment in the AWM.

\subsection{Benchmarking Against Commitment}

Conducting optimal policy under commitment may not be feasible for the policymaker as it becomes problematic for the outside world to monitor policy whenever it conditions on a large set of states. For the sake of completeness, we highlight the role for more complicated commitment policy in the AWM. Commitment is by definition enhancing over simple rules (given that it potentially encompasses all states) and should also (though to a lesser extent) dominate the discretionary solution. We found pronounced difficulties in solving for the fully optimal commitment solution and considered that our results for that solution may not be particularly robust to the way the transmission of structural shocks has been identified. ${ }^{19}$ We resort to an approximation of the first best commitment solution. Giannoni and Woodford (2003) have shown theoretically that the optimal commitment policy can be approximated closely by a generalized Taylor rule which in addition to the contemporaneous feedback contains enough lags and expectational leads of the target variables themselves. While for smaller more forward-looking models the number of lags and leads needed is rather contained, apparently this is not the case for a model

${ }^{19}$ As regards the computation, the AWM even after linearization is nearly non-stationary due to the "exogenous" processes. For numeric reasons, it is impossible to solve the model augmented by the costates as in Dennis (2001) by AIM. When resorting to Sims' (2001) procedure, the determinacy conditions yield one loose endogenous error, rendering the equilibrium indeterminate. As Lubik and Schorfheide (2003) highlight, in that case not only might sunspot shocks effect the economy, but also the transmission of structural shocks through the model is not uniquely determined. We overrode the indeterminacy issue and used the identification provided by Sims. This resulted in discretion being $20 \%$ to $70 \%$ worse than commitment. These values are larger than in the literature and somewhat surprising given the limited role of expectations in the AWM. Since these results suffer from the identification problem mentioned above, we do not consider these to be reliable. For completeness, we nevertheless report these results in Appendix F. 
incorporating a complicated lag structure like the AWM. For one set of preferences, Table 7 displays the losses involved when committing to an optimized rule with a total of ten lags and three expectational leads - and hence 41 free parameters. The final row reports results for four lags and six leads. As is apparent, the gains over optimal discretionary

Table 7: Pseudo-Commitment ${ }^{a}$

\begin{tabular}{||c|c||c|c|c|c||c||}
\hline \hline$\lambda$ & $\gamma$ & $\sigma_{\text {gap }}$ & $\sigma_{\pi}$ & $\sigma_{\Delta r}$ & $\sigma_{r}$ & Loss \\
\hline \hline \multicolumn{7}{||c||}{$\bar{\sigma}_{\Delta r}$ free } \\
\hline 0.00 & 0.50 & 1.71 & 1.02 & 0.46 & 2.17 & 1.15 \\
\hline 0.10 & 0.50 & 1.58 & 1.02 & 0.53 & 2.22 & 1.42 \\
\hline 0.33 & 0.50 & 1.40 & 1.01 & 0.71 & 2.50 & 1.94 \\
\hline 1.00 & 0.50 & 1.19 & 1.01 & 1.09 & 3.14 & 3.04 \\
\hline 3.00 & 0.50 & 0.96 & 1.01 & 1.73 & 4.10 & 5.26 \\
\hline \hline 1.00 & 0.50 & 1.19 & 1.01 & 1.09 & 3.15 & 3.03 \\
\hline
\end{tabular}

${ }^{a}$ The Table reports results for pseudo-commitment. The rules include 10 lags and 3 expectational leads of the target variables gap, $\pi, \Delta r$. The exception is the last line, which entertains 4 lags and 6 leads.

policy are mild at best. Even complicated rules as these do not improve greatly upon the discretionary policy and fall far short of the commitment results reported in appendix $\mathrm{F}$ that appear when using Sims' (2001) identification of endogenous and exogenous shocks. In the framework of the AWM, with a large set of states and high intrinsic persistence paired with limited forward-lookingness, even such highly complicated rule-based commitment policy does not improve considerably on the discretionary outcome. To us, this highlights once more that in such an environment, it is extremely important to capture the information content in all state variables. ${ }^{20}$ For a comparison, Finan and Tetlow (1999) report for the more forward-looking FRB/US model, which features roughly 300 equations, that a simple optimized contemporaneous Taylor rule is between $14 \%$ to $25 \%$ worse than the optimal commitment solution. If we take the pseudo-commitment rules as close enough approximation to fully optimal commitment, results for the AWM range at the upper limit of this interval owing to the strong persistence and limited forward-

${ }^{20}$ Optimizing 41 parameters already turns out to be computationally expensive. As the above exercise serves mainly illustrative purposes, we report results only for one set of preferences. In addition, we refrain from approximating commitment policy more closely by adding more leads and lags to the rules. Accordingly, we do not claim that the results reported constitute the global optimum under commitment but rather term the long rules "pseudo-commitment." 
lookingness of the model. As Table 8 illustrates, optimal simple rules can increase losses by roughly $27 \%$ relative to pseudo-commitment. Again, that the deterioration from using

Table 8: Relative Losses for $\gamma=0.5^{a}$

\begin{tabular}{||c||c||c|c|c|c||}
\hline \hline Rule & \% Loss & $\sigma_{g a p}$ & $\sigma_{\pi}$ & $\sigma_{\Delta r}$ & $\sigma_{r}$ \\
\hline \hline \multicolumn{5}{||c||}{ Strict inflation targeter, $\lambda=0$} \\
\hline Contemporaneous & - & 1.93 & 1.17 & 0.64 & 2.83 \\
\hline Lagged & 6.37 & 2.09 & 1.20 & 0.67 & 2.92 \\
\hline Forecast-Based & -14.64 & 1.82 & 1.08 & 0.58 & 2.58 \\
\hline Discretion & -26.11 & 1.70 & 1.02 & 0.47 & 2.19 \\
\hline Pseudo-Commitment & -26.75 & 1.71 & 1.02 & 0.46 & 2.17 \\
\hline
\end{tabular}

\begin{tabular}{||c||c||c|c|c|c||}
\hline \hline \multicolumn{5}{||c||}{ Flexible inflation targeter, $\lambda=1$} \\
\hline Contemporaneous & - & 1.31 & 1.10 & 1.39 & 3.81 \\
\hline Lagged & 21.9 & 1.58 & 1.13 & 1.39 & 3.72 \\
\hline Forecast-Based & -9.02 & 1.28 & 1.03 & 1.30 & 3.61 \\
\hline Discretion & -21.6 & 1.19 & 1.01 & 1.09 & 3.12 \\
\hline Pseudo-Commitment & -21.6 & 1.19 & 1.01 & 1.09 & 3.14 \\
\hline \hline
\end{tabular}

\begin{tabular}{||c||c||c|c|c|c||}
\hline \hline \multicolumn{5}{||c||}{ Given Policy Rules, $\lambda=0(\lambda=1)$} \\
\hline Taylor & $70.9(75.4)$ & 2.03 & 1.23 & 1.53 & 2.33 \\
\hline Gerdesmeier-Roffia & $32.5(103.3)$ & 2.41 & 1.36 & 0.68 & 2.44 \\
\hline Clarida et al. & $27.9(88.1)$ & 2.30 & 1.39 & 0.39 & 1.70 \\
\hline Gerlach-Schnabel & $1.3(34.0)$ & 1.90 & 1.18 & 0.63 & 1.85 \\
\hline
\end{tabular}

a \%Loss is the percentage increase in loss relative to the loss under the respective optimized simple contemporaneous rule for $\lambda=0$ and $\lambda=1$, respectively. The Table refers to the results with a punishment $\gamma=0.5$ on interest rate change variability.

less complicated rules is at times sizeable can be attributed to the fact that the AWM is very much persistent.

A conclusion emerging from the results of this section is that the Central Bank if it is willing to condition its policy on the AWM framework should take all available information into account even if this results in very complicated interest rate rules. We next turn to illustrate the gains to forward-looking policy in a framework as the AWM. 


\section{Optimal Forecast Horizons}

The more forward-looking a model, the more agents take into account both current and future policy conditions, thereby in effect reducing the importance of policy lags and the uncertainty surrounding them. In contrast, as Batini and Nelson (2001) highlight, in backward-looking models, i.e., in the absence of an expectations channel or with a large degree of intrinsic persistence, policy lags may be longer thereby leading to an optimal forecast horizon farther into the future. With too long a forecast horizon, however, indeterminacy can occur for any value of the feedback parameters as argued by Batini et al. (2003). ${ }^{21}$ We turn to the issue of optimal forecast horizons in due course focusing on the benefits of choosing an optimal inflation forecast horizon first (which has been most intensively emphasized in the literature so far).

\subsection{Optimal Forecast Horizons for Inflation}

We minimize loss function (1) with respect to the feedback parameters $\rho, \alpha$ and $\beta$ and discretely for the forecast horizon $\theta$ for a policy-maker who credibly implements the forecast based rule

$$
r_{t}=\rho r_{t-1}+\alpha E_{t} \pi_{t+\theta}+\beta g a p_{t}, \theta \in\{1,2, \ldots, 16\}
$$

As we seek to derive conclusions closest to the current policy regime as possible and in order not to overload the current paper, we focus exclusively on the case with an empirical upper bound on interest change variability. Table 9 summarizes the results, which figure 7 illustrates graphically. The optimal forecast horizon for a strictly inflation targeting central bank is $\theta=10$ quarters. The optimal forecast horizon is associated with a loss only $1.2 \%$ larger than the corresponding loss under discretion and full information, compared to a $16.5 \%$ larger loss with a conventional one year forecast horizon. A sizeable portion of model information can thus be incorporated by means of inflation forecastbased rules with an optimally chosen horizon. For a policy-maker who weights output gap stabilization, $\lambda=\frac{1}{3}$, the optimal horizon, $\theta$, is at 11 quarters. The loss in distance in

${ }^{21}$ With constant interest rates, the AWM is stationary, but the equilibrium is indeterminate - there are an infinite number of paths on which the economy can converge to the stable growth path. In the stationary environment, as the forecast horizons tend to infinity, the expectations of inflation and the output gap converge to their unconditional expectations, zero. Interest rates then are exogenous and constant. 
Table 9: Optimized Policy Under Different Forecast Horizons for Inflation ${ }^{a}$

\begin{tabular}{|c|c|c|c|c|c|c|c|}
\hline$\theta$ & $\rho$ & $\alpha$ & $\beta$ & $\sigma_{g a p}$ & $\sigma_{\pi}$ & $\sigma_{r}$ & Loss \\
\hline \multicolumn{8}{|c|}{$\lambda=0$} \\
\hline 2 & 0.67 & 0.74 & 0.28 & 1.89 & 1.14 & 2.60 & 1.29 \\
\hline 6 & 0.69 & 1.69 & 0.16 & 1.75 & 1.04 & 2.53 & 1.07 \\
\hline 8 & 0.73 & 2.29 & 0.15 & 1.72 & 1.02 & 2.49 & 1.03 \\
\hline 12 & 0.82 & 3.94 & 0.12 & 1.71 & 1.01 & 2.46 & 1.03 \\
\hline 16 & 0.97 & 9.24 & 0.00 & 1.73 & 1.02 & 2.44 & 1.04 \\
\hline \multicolumn{8}{|c|}{$\lambda=\frac{1}{3}$} \\
\hline 2 & 0.61 & 0.66 & 0.36 & 1.83 & 1.15 & 2.42 & 2.43 \\
\hline 6 & 0.63 & 1.43 & 0.30 & 1.67 & 1.05 & 2.37 & 2.03 \\
\hline 8 & 0.64 & 1.89 & 0.33 & 1.62 & 1.03 & 2.34 & 1.94 \\
\hline 12 & 0.65 & 2.93 & 0.39 & 1.60 & 1.03 & 2.30 & 1.91 \\
\hline 16 & 0.64 & 4.67 & 0.37 & 1.60 & 1.04 & 2.24 & 1.93 \\
\hline \multicolumn{8}{|c|}{$\lambda=1$} \\
\hline 2 & 0.58 & 0.61 & 0.39 & 1.82 & 1.16 & 2.37 & 4.63 \\
\hline 6 & 0.60 & 1.31 & 0.34 & 1.66 & 1.06 & 2.33 & 3.87 \\
\hline 8 & 0.61 & 1.72 & 0.39 & 1.61 & 1.04 & 2.31 & 3.68 \\
\hline 12 & 0.60 & 2.57 & 0.45 & 1.59 & 1.04 & 2.27 & 3.60 \\
\hline 16 & 0.58 & 3.88 & 0.44 & 1.59 & 1.04 & 2.21 & 3.61 \\
\hline
\end{tabular}

${ }^{a}$ Varying inflation forecast horizons, $\theta$. The Table reports results for policy rule (7). An empirical upper bound on interest rate change variability is imposed, $\bar{\sigma}_{\Delta r}=0.569$. Details on other combinations are available.

loss relative to the optimal policy under discretion reduces from $23.8 \%$ to $6 \%$. Similarly with $\lambda=1$, a forecast horizon of $\theta=12$ is optimal with a sizeable reduction in loss relative to optimal discretionary policy. The optimal forecast horizons hence are rather long and gains are substantial.

The optimality of long forecast horizons squares with Batini and Nelson (2001). While these long horizons are optimal for the AWM, the mere fact that more and more information (states as well as model structure) is implemented into policy poses the question how these rules will perform under model uncertainty. While we leave this question for future research, we note that most of the gains are realized already within a forecast horizon of one and a half years (6 quarters) (see figure 7 ) which, we conjecture, will be less prone to 

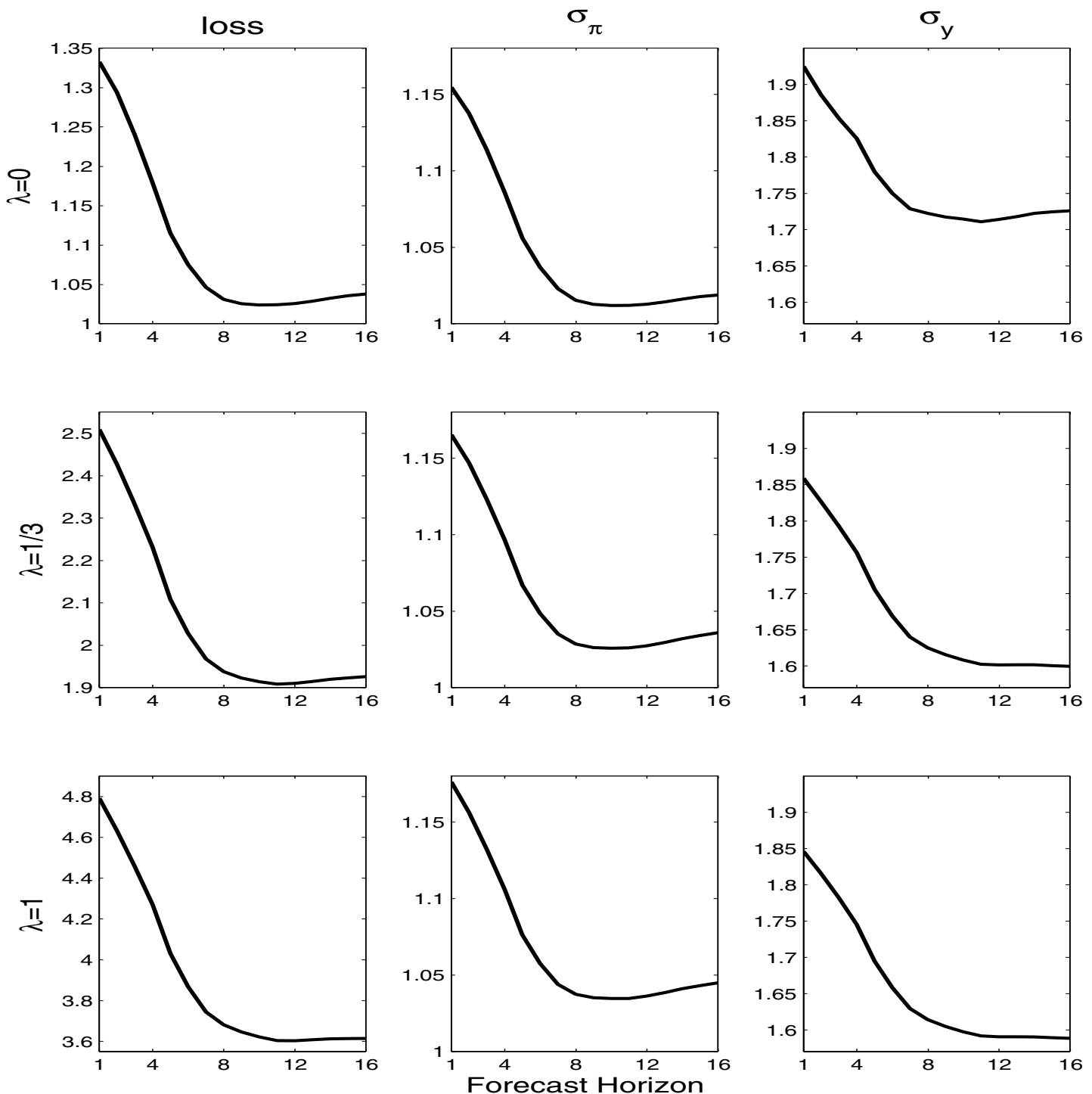

Figure 7: Optimal forecast horizon, $\theta$, for the inflation rate when the horizon for the output gap, $\kappa=0$. From left column to right column: loss, standard deviation of inflation and standard deviation of the output gap. From top to bottom: increasing weight on stabilization of the output gap, $\lambda=0, \frac{1}{3}, 1$. An empirical bound on interest rate change variability is imposed.

model uncertainty. A policymaker caring for stabilization is well advised in the framework of the AWM to react to excessive inflation far into the future and not to react to shocks with only a temporary effect on inflation. This high degree of forward-lookingness on behalf of the policymaker sharply contrasts with Levin et al. (2003) who find a horizon $\theta$ of one to four quarters optimal. Again, this may be due to the substantially stronger forward-lookingness embedded in their models and the lower intrinsic persistence. 


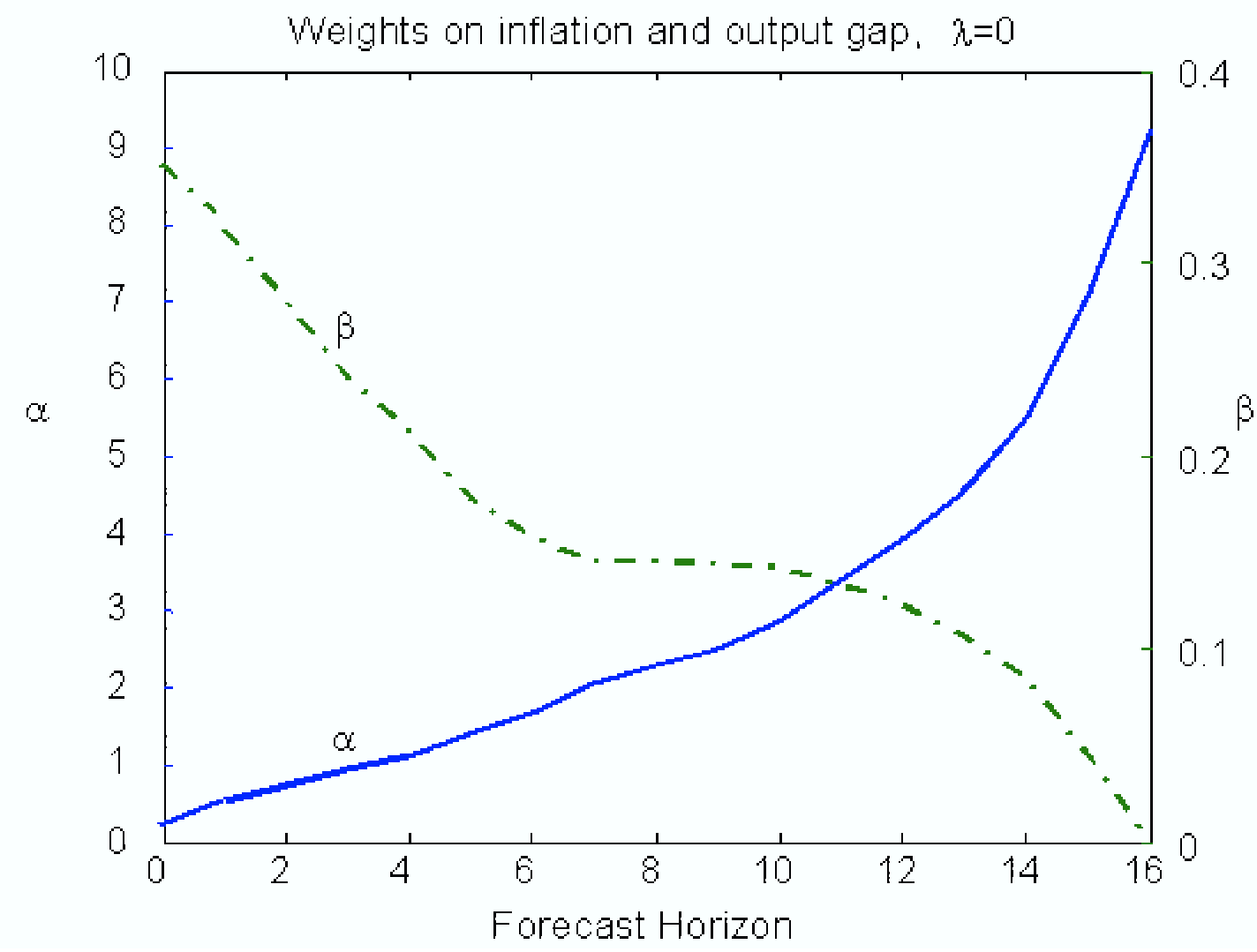

Figure 8: Weights on the expected inflation term (solid line) and the output gap term (dotted line) for strict inflation targeting preferences for varying forecast horizons for inflation.

Focusing on the results for the varying inflation forecast horizons for the strict inflation target, $\lambda=0$, figure 8 highlights that the output gap looses its value as an indicator variable for the inflation target relative to the expected inflation measure as the forecast horizon increases. The optimal weight, $\beta$ is reduced to roughly half its optimal value under the contemporaneous rule at a forecast horizon $\theta>5$ and essentially reduced to zero as the inflation forecast horizon increases further.

We have illustrated that the persistence of the AWM asks for rather anticipative policy. Forecast horizons for inflation longer than two years should be emphasized in policy making. We next turn to a joint maximization of both forecast horizons. 


\subsection{Optimal Forecast Horizons for both the Output Gap and the Inflation Rate}

Adding to the information provided in the previous section, policymakers may seek to optimally target both inflation and the output gap at lead horizons. The rules we thus consider in this section are

$$
r_{t}=\rho r_{t-1}+\alpha E_{t} \pi_{t+\theta}+\beta E_{t} g a p_{t+\kappa}, \theta \in\{1,2, \ldots 16\}, \kappa \in\{1,2, \ldots 8\} .
$$

In order to highlight the gains associated with the optimal choice of the output gap forecast horizon, for each value of $\lambda \in\left[0, \frac{1}{3}, 1\right]$, Table 11 reports the optimal rules under the optimal horizon for inflation and output gap forecasts along with the results for maximizing the inflation forecast horizon only and the results for optimal discretionary policy.

Table 10: Optimized Forecast Horizons for Output Gap and Inflation ${ }^{a}$

\begin{tabular}{|c|c|c|c|c|c|c|c|c|}
\hline$\theta$ & $\kappa$ & $\rho$ & $\alpha$ & $\beta$ & $\sigma_{\text {gap }}$ & $\sigma_{\pi}$ & $\sigma_{r}$ & Loss \\
\hline \multicolumn{9}{|c|}{$\lambda=0$} \\
\hline 10 & 0 & 0.78 & 2.86 & 0.14 & 1.71 & 1.01 & 2.47 & 1.02 \\
\hline 10 & 4 & 0.84 & 2.74 & 0.24 & 1.67 & 1.01 & 2.44 & 1.02 \\
\hline \multicolumn{5}{|c|}{ Discretion } & 1.66 & 1.01 & 2.39 & 1.01 \\
\hline \multicolumn{9}{|c|}{$\lambda=\frac{1}{3}$} \\
\hline 12 & 0 & 0.65 & 2.93 & 0.39 & 1.60 & 1.03 & 2.30 & 1.91 \\
\hline 11 & 5 & 0.86 & 1.50 & 0.76 & 1.50 & 1.03 & 2.27 & 1.81 \\
\hline \multicolumn{5}{|c|}{ Discretion } & 1.49 & 1.03 & 2.22 & 1.80 \\
\hline \multicolumn{9}{|c|}{$\lambda=1$} \\
\hline 12 & 0 & 0.60 & 2.57 & 0.45 & 1.59 & 1.04 & 2.27 & 3.60 \\
\hline 16 & 7 & 0.96 & 1.99 & 1.55 & 1.48 & 1.05 & 2.22 & 3.28 \\
\hline \multicolumn{5}{|c|}{ Discretion } & 1.47 & 1.05 & 2.18 & 3.27 \\
\hline
\end{tabular}

${ }^{a}$ Optimal forecast horizon for inflation, $\theta$, and the output gap, $\kappa$. The Table reports results for policy rule (8). An empirical upper bound on interest rate change variability, $\bar{\sigma}_{\Delta r}=0.569$, is imposed. The first row for each weight on the output gap, $\lambda$, reports optimal forecast horizons for inflation, when $\kappa=0$. The second rows report the result of joint optimization over $\theta, \kappa$ and the feedback parameters. The third rows benchmark against the discretionary policy. Details on other combinations are available.

Perhaps not much surprisingly, the largest reduction of losses is achieved when output 
is a target variable - and the gains are the larger the more a policymaker is interested in stabilizing the real side of the economy. For a strict inflation target, in contrast, the gain of the optimized output gap forecast horizon is almost nil. Most of the reduction of losses is achieved by an optimal choice of forecast horizon for inflation alone. The most sizeable reductions of losses when altering $\kappa$ appear within a more conventional window of one to two years.

Overall, these results highlight that with optimally chosen forecast horizons of roughly two to three years for inflation and one to two years for the output gap, the central bank by implementing a credible rule can almost achieve the outcome of fully optimal discretionary policy - recalling Table 8 it thereby also achieves good performance as benchmarked against the pseudo-commitment policy.

In so far as a simple policy remains credible even at these long forecast horizons and in so far as simple policy would be easier to conduct than fully optimal (and thereby, as we argued above, almost necessarily discretionary) policy, the central bank will have to emphasize that policy should be immensely focused on future conditions. In addition, in so far as model uncertainty prevails, simple forecast based rules may be more robust to model uncertainty than fully optimal rules - we leave that issue for future examination.

While the AWM implies much longer inflation forecast horizons than the forwardlooking models discussed, e.g., in Levin et al. (2003) our results for the optimal output gap forecast horizon is in line with their study - although at the upper bound of their results. Summarizing, above results again highlight, that optimal policy in the AWM should emphasize forward-looking elements and should incorporate as much of the information in the model structure and the state vector as possible even at the loss of simplicity.

\section{Conclusions}

Understanding optimal policy rules for the euro area is (and will be of) crucial importance for policymakers. This paper contributed to the debate by evaluating the conduct of optimal monetary policy in a medium-scale model of the euro area, the AWM.

We illustrate that the model features a high degree of intrinsic persistence, i.e., persistence not introduced by monetary policy itself. In addition, the AWM is more traditional in attributing only a limited role for forward-looking expectations and explicit micro- 
foundations. In evaluating optimized generalized Taylor (1993) rules with interest rate smoothing and in benchmarking them against fully optimal policy under discretion and commitment, we obtain the following results.

We highlight that in contrast to small-scale models or the forward-looking New Keynesian paradigm models, policymakers may be advised to base their decisions on a broad information set. In line with recent analysis by Côté et al. (2002) for example, we find that optimal policy per se calls for rather mild instrument smoothing: first-difference or super-inertial rules are not, for example, stabilizing in this model or, we suspect, in this class of models. These results square with the recent analysis of Levin and Williams (2003) who illustrate that the degree of interest rate smoothing is the crucial ingredient in the Taylor rule that is prone to be destabilizing under model uncertainty. In the rather backward looking and highly persistent framework of the AWM, optimal forecast horizons are also relatively long: up to two and a half years for the inflation forecast and one year for the output gap forecast for a strict inflation target and longer whenever policy puts more emphasis on real stabilization.

The analysis presented here is of course conditional on a single model economy albeit one which may well be representative of policy and forecasting models used in many central banks. Because conclusions about the optimal conduct of policy are often sensitive to the dynamic specification of models, it would be prudent to analyze the predicted performance of a contemplated rule under alternative modelling assumptions. We understand this paper to mark one end of possible modelling approaches for the euro area. Whenever central banks consider a highly persistent model with a private sector that is rather backward-looking sufficiently probabilistic, optimal policy should be very much concerned with future conditions and should seek to incorporate a broad information set into the policy making process.

Future research should put the results reported herein into the context of other euro area model paradigms and should seek to extract robust policy conclusions - from as diverse a model space as possible. Assessing the costs and gains of robust policy making would certainly be a valuable contribution in advancing the optimal monetary policy discussion for the euro area. 


\section{References}

Anderson, G. and Moore, G. (1985). A Linear Algebraic Procedure for Solving Linear Perfect Foresight Models. Economics Letters, 17(3):247-252.

Ball, L. (1999). Efficient Rules Monetary Policy. International Finance, 2:63-83.

Batini, N. and Haldane, A. (1999). Forward-Looking Rules for Monetary Policy. In Taylor, J. B., editor, Monetary Policy Rules. Chicago University Press, Chicago.

Batini, N. and Nelson, E. (2001). Optimal Horizons for Inflation Targeting. Journal of Economic Dynamics and Control, 25:891-910.

Bernanke, B. and Woodford, M. (1997). Inflation Forecasts and Monetary Policy. Journal of Money Credit and Banking, 24:653-684.

Binder, M. and Pesaran, H. (1995). Multivariate Rational Expectations Models and Macroeconomic Modeling: A Review and Some New Results. In Pesaran, H. and Wickens, M., editors, Handbook of Applied Econometrics. Blackwell Publishers.

Blanchard, O. and Kahn, C. (1980). The Solution of Linear Difference Models under Rational Expectations. Econometrica, 48(5):1305-1311.

Brainard, W. (1967). Uncertainty and the Effectiveness of Policy. American Economic Review, Papers and Proceedings, 57(2):411-425.

Clarida, R., Gali, J., and Gertler, M. (1998). Monetary Policy Rules in Practice. Some International Evidence. European Economic Review, 42(6):1033-1067.

Clarida, R., Gali, J., and Gertler, M. (1999). The Science of Monetary Policy: A New Keynesian Perspective. Journal of Economic Literature, 37:1661-1707.

Clarida, R., Gali, J., and Gertler, M. (2000). Monetary Policy Rules and Macroeconomic Stability: Evidence and Some Theory. Quarterly Journal of Economics, 115:147-180.

Coenen, G. (2003). Inflation Persistence and Robust Monetary Policy Design. European Central Bank Working Paper Series 290, Frankfurt: European Central Bank.

Côté, D., Kuszczak, J., Lam, J. P., Ying, L., and St-Amant, P. (2002). The Performance and Robustness of Simple Monetary Policy Rules in Models of the Canadian Economy. Technical Report 92, Bank of Canada.

Cukierman, A. (1990). Why Does the Fed Smooth Interest Rates? In (Ed.), M. B., editor, Monetary Policy of the Fed's 75 Anniversary. Kluwer Academic Publishers.

Dennis, R. (2001). Optimal Policy in Rational-Expectations Models: New Solution Algorithms. Working Papers in Applied Economic Theory 2001-09, San Francisco: Federal Reserve Bank of San Francisco.

Detken, C., Dieppe, A., Henry, J., Marin, C., and Smets, F. (2002). Determinants of the Effective Real Exchange Rate of the Synthetic Euro. Australian Economic Papers, 41(4):404-436.

Dieppe, A. and Henry, J. (2004). The Euro Area Viewed as a Single Economy: How Does It Respond to Shocks? Economic Modelling. forthcoming. 
Dieppe, A., Henry, J., and McAdam, P. (2003). Labour Market Dynamics in the Euro Area: A Model-Based Sensitivity Analysis. In Semmler, W., editor, Monetary Policy and the Labor Market in the U.S., The Euro-Area and Japan: A Conference in Honor of James Tobin. Routledge Press.

ECB (2001). A Guide to Eurosystem Staff Macroeconomic Projection Exercises. European Central Bank, Frankfurt.

Fagan, G., Henry, J., and Mestre, R. (2001). An Area-Wide Model (AWM) for the Euro Area. ECB Working Paper Series 42, Frankfurt: European Central Bank.

Finan, F. S. and Tetlow, R. (1999). Optimal Control of Large, Forward-Looking Models: Efficient Solutions and Two Examples. Finance and Economics Discussion Series 1999-51, Washington: Board of Governors of the Federal Reserve System.

Fuhrer, J. (1997). The (Un)importance of Forward-Looking Behaviour in Price Specifications. Journal of Money, Credit and Banking, 29(3):338-350.

Fuhrer, J. and Estrella, A. (2002). Counterfactual Implications of a Class of RationalExpectations Models. American Economic Review, 92(4):1010-1028.

Gerdesmeier, D. and Roffia, B. (2003). Empirical Estimates of Reaction Functions for the Euro Area. ECB Working Paper Series 206, Frankfurt: European Central Bank.

Gerlach, S. and Schnabel, G. (2000). The Taylor Rule and Interest Rates in the EMU Area. Economics Letters, 67:165-171.

Giannoni, M. P. and Woodford, M. (2003). Optimal Interest-Rate Rules: I. General Theory. NBER Working Paper Series: w9419, Cambridge, Mass.: NBER.

Hansen, L. P. and Sargent, T. J. (1997). Recursive Models of Linear Dynamic Economies, Ch. 9. Stanford University. Available at http://www.stanford.edu/ sargent/hansen.html.

Levin, A., Wieland, V., and Williams, J. (1999). Robustness of Simple Monetary Policy Rules under Model Uncertainty. In Taylor, J. B., editor, Monetary Policy Rules. University of Chicago Press, Chicago.

Levin, A., Wieland, V., and Williams, J. (2003). The Performance of Forecast-Based Monetary Policy Rules under Model Uncertainty. American Economic Review, 93(3):622645.

Levin, A. T. and Williams, J. C. (2003). Robust Monetary Policy with Competing Reference Models. Journal of Monetary Economics, 50:945-975.

Lubik, T. A. and Schorfheide, F. (2003). Computing Sunspot Equilibria in Linear Rational Expectations Models. Journal of Economic Dynamics and Control, 28(2):273-286.

Mankiw, N. G. and Reis, R. (2003). What Measure of Inflation Should a Central Bank Target. Journal of European Economic Association, 1(5):1058-1086.

McAdam, P. and Hughes Hallett, A. J. (1999). Non Linearity, Computational Complexity and Macro Economic Modelling. Journal of Economic Surveys, 13(5):577-618.

McAdam, P. and Mestre, R. (2003). Evaluating Macro-Modeling Systems: An Application to the Area Wide Model. Downloadable from http://www.federalreserve.gov/ events/cbw/mcadam_mestre_aug30.pdf. 
McAdam, P. and Morgan, J. (2003). The Monetary Transmission Mechanism at the Euro Area Level: Issues and Results Using Structural Macroeconomic Models. In Angeloni et al. , editor, Monetary Policy Transmission in the Euro Area. Cambridge University Press, Cambridge.

Orphanides, A. (2001). Monetary Policy Rules Based on Real-Time Data. American Economic Review, 91(4):964-985.

Rotemberg, J. J. and Woodford, M. (1998). An Optimization-Based Econometric Framework for the Evaluation of Monetary Policy : Expanded Version. NBER Working Paper 233.

Sims, C. A. (2001). Solving Linear Rational Expectations Models. Computational Economics, 20:1-20.

Svensson, L. E. (1997). Inflation Forecast Targeting: Implementing and Monitoring Inflation Targets. European Economic Review, 41:1111-1146.

Svensson, L. E. O. (1999a). Inflation Targeting as a Monetary Rule. Journal of Monetary Economics, 43:607-654.

Svensson, L. E. O. (1999b). Inflation Targeting: Some Extensions. Scandinavian Journal of Economics, 101(3):337-361.

Svensson, L. E. O. (2000). Open-Economy Inflation Targeting. Journal of International Economics, 50:155-183.

Taylor, J. B. (1993). Discretion Versus Policy Rules in Practice. Carnegie-Rochester Conference Series on Public Policy, 39:195-214. 


\section{A Linearization and Parameter Choices}

By means of the error correction mechanisms build into the model equations, the nonstationary system converges to a balanced growth path, see Fagan et al. (2001). This ensures that suitably defined ratios of real variables and ratios of nominal variables ultimately converge to a unique steady state value. ${ }^{22}$ We provide two examples for the linearization in which we neglect constants and error terms for convenience. In the following, capital letters (XTD) mark variables in the original non-linear model file described in Fagan et al. (2001), small letters (xtd) mark well-defined steady state ratios. Italic letters $(x t d)$ mark variables in percentage deviation from steady state as they appear in the linearized model version. The first example is a log-linear equation defining the export deflator, XTD. In the original model:

$$
\begin{array}{r}
\Delta \log \mathrm{XTD}_{t}=\phi_{1} \Delta \log \mathrm{XTD}_{t-1}+\phi_{2} \Delta \log \mathrm{YED}_{t}+\phi_{3} \Delta \log \mathrm{EEN}_{t}+\phi_{4} \Delta \log \mathrm{MTD}_{t-1} \\
+\phi_{5}[0.7 \log \mathrm{XTD} / \mathrm{YED}+0.3 \log \mathrm{XTD} /(\mathrm{YWD} \mathrm{EEN})]_{t-1},
\end{array}
$$

where the $\phi_{i}$ are parameters, YED is the GDP deflator, EEN is the nominal effective exchange rate, MTD is the import deflator and YWD is the world GDP deflator. Furthermore, let YFD be the GDP at factor cost deflator. We define price ratios $x t d=\frac{X T D}{Y F D}$, yed $=\frac{\text { YED }}{\text { YFD }}, \operatorname{mtd}=\frac{\text { MTD }}{\text { YFD }}$ and ywd $=\frac{\text { YWD EEN }}{\text { YFD }}$. In addition, we define the following inflation rates: pieen $=\Delta \log$ EEN, pixtd $=\Delta \log$ XTD, piyed $=\Delta \log$ YED and pimtd $=\Delta \log$ MTD. All these lower case price ratios and inflation rates have well-defined steady states. Next we express variables in percentage (log) deviation from steady state as, for instance, $x t d=\frac{\mathrm{xtd}_{t}-\overline{\mathrm{xtd}}}{\overline{\mathrm{xtd}}} \simeq \log \mathrm{xtd}_{t}-\log \overline{\mathrm{xtd}}$ and pimtd $=\operatorname{pimtd}_{t}-\overline{\operatorname{pimtd}}$.

The linear approximation of (9) about the balanced growth path, which we are heading for, then reads as

pixtd $_{t}=\phi_{1}$ pixtd $_{t-1}+\phi_{2}$ piyed $_{t}+\phi_{3}$ pieen $_{t}+\phi_{4}$ pimtd $_{t-1}+\phi_{5}[\text { xtd }-0.7 \text { yed }-0.3 y w d]_{t-1}$.

Elsewhere in the model file for the linearized model, we relate inflation rates to levels of the respective variables. An example of this would be pixtd $d_{t}=x t d_{t}-x t d_{t-1}+p i y f d_{t}$. The second example of the linearization comprises the linear GDP identity

$$
\mathrm{YER}=\mathrm{PCR}+\mathrm{GCR}+\mathrm{ITR}+\mathrm{TBR}+\mathrm{SCR},
$$

where YER is real GDP, PCR is private consumption, GCR is government consumption, ITR is investment, TBR is the real trade balance and SCR is the variation of stocks. We normalize these variables by potential output, YET, in order to obtain suitable ratios yer $=\frac{\mathrm{YER}}{\mathrm{YET}}, \mathrm{pcr}=\frac{\mathrm{PCR}}{\mathrm{YET}}, \mathrm{gcr}=\frac{\mathrm{GCR}}{\mathrm{YET}}, \mathrm{itr}=\frac{\mathrm{ITR}}{\mathrm{YET}}, \mathrm{tbr}=\frac{\mathrm{TBR}}{\mathrm{YET}}$, and $\mathrm{scr}=\frac{\mathrm{SCR}}{\mathrm{YET}}$. Again, all small case variables will ultimately converge to their steady state value. Rewriting (10), we have

$$
\text { yer }=\text { pcr }+ \text { gcr }+ \text { scr }+ \text { itr }+ \text { tbr. }
$$

\footnotetext{
22 More exactly, this refers to the non-stochastic balanced growth path. Accordingly, speaking of the steady state values of certain variables, henceforth we shall mean the constant values of these variables associated with the non-stochastic balanced growth path.
} 
Defining percentage deviations from steady state as $y r_{t}=\frac{\mathrm{yer}_{t}-\overline{\mathrm{yer}}}{\overline{\mathrm{yer}}}$ and similarly for the other variables, equation (11) can be approximated around steady state as

$$
y e r=\frac{\overline{\mathrm{pcr}}}{\overline{\mathrm{yer}}} p c r+\frac{\overline{\mathrm{gcr}}}{\overline{\mathrm{yer}}} g c r+\frac{\overline{\mathrm{itr}}}{\overline{\mathrm{yer}}} i t r+\frac{\overline{\mathrm{tbr}}}{\overline{\overline{\mathrm{yer}}}} \operatorname{tbr}+\left(1-\frac{\overline{\mathrm{pcr}}}{\overline{\mathrm{yer}}}-\frac{\overline{\mathrm{gcr}}}{\overline{\mathrm{yer}}}-\frac{\overline{\mathrm{itr}}}{\overline{\mathrm{yer}}}-\frac{\overline{\mathrm{tbr}}}{\overline{\mathrm{yer}}}\right) s c r .
$$

Apparently, for instance the steady state share of private consumption in GDP, $\frac{\overline{\mathrm{pcr}}}{\overline{\mathrm{yer}}}=\frac{\overline{\mathrm{PCR}}}{\mathrm{YER}}$, appears in (12). Further steady state ratios will appear in other equations. Most of these can be solved for by using the primitives of the model, such as population growth, the inflation target and the original non-linear model equations. The non-linearity of the original model, however, prevents us from obtaining closed form solutions for all the steady state ratios. In these cases we resort to ratios obtained from long-run simulations of the original model implemented in TROLL. Apart from some fiscal ratios, we need to set $\overline{\frac{\mathrm{PCR}}{\text { YER }}}$ and $\overline{\overline{\mathrm{GCR}}}$, the ratios of world GDP to home GDP and the ratios of world and commodity prices to home prices. While these values will depend on the path that the economy takes and hence especially on the calibration for the exogenous variables, our analysis did not prove sensitive to minor perturbations in the steady state ratios chosen. All other steady state ratios appearing in the model are solved for in a model-consistent fashion using the model-equations.

The current non-linear AWM version assumes that the levels of government consumption, foreign GDP, foreign prices and commodity prices as well as trend factor productivity and population on the balanced growth path grow at constant, exogenous rates. We need to endogenize these series for the solution methods described below. The endogenization introduces unitary roots into the companion matrix of the model solution as there is no mechanism within the model to force ratios involving these variables back to an endogenously defined steady state. For example, an exogenous rate of government consumption implies, and is expressed as, $\Delta g c r=$ piyet, where piyet is the growth rate of potential output in deviation from steady state. As there is no mechanism to force GCR after a potential shock back to its steady state level (i.e. to the level the model is currently calibrated to), this formulation introduces a unitary root. Since both, the calculation of variances for relevant model variables from the companion form as well as the algorithms to compute optimal discretionary policy require stationary models, we 'stationarize' the model by rewriting the respective random walk equations as highly persistent autoregressive processes in order to avoid numerical problems. For above example, this is $g c r_{t}=(1-a) g c r_{t-1}+$ piyet, where $a=1 . e-6$. Note that this introduces only minor changes to the model, as effects thereof will only materialize in the very long run. Similarly, we proceed to eliminate the unitary roots introduced by the other five exogenous variables. Apart from the estimated parameter values, a few key assumptions about steady state values are taken from the calibration of the non-linear model version. We report these up to two significant digits: trend unemployment rate $(9.1 \%)$, quarteron-quarter inflation rate $(0.49 \%)$, annualized quarterly nominal interest rate (499 bps), quarterly output growth rate $(0.57 \%)$, share of real government consumption in trend output (14\%). 
The linear version has been rigorously benchmarked against the original model. We found hardly any sizeable differences between the impulse-responses generated by the two models.

\section{B First-order Form}

In order to save on computation time, we transform the model into containing lags and leads of one period only (see for example Binder and Pesaran, 1995, for how to anchor leads and lags in such a representation). This explains that while the linear model contains only 67 'core equations', ${ }^{23}$ it features 145 equations defining lags and leads of other state variables. $^{24}$ Together with 14 white noise shocks to behavioral equations, there are 226 equations. $^{25}$

\section{Empirical Variance Covariance Matrix and Data}

To obtain an estimate $V(u)$ of the variance covariance matrix of the shocks we decided to use the series in the AWM data set from 1980q1 to 2000q4. The time series pertaining to the endogenous variables carrying shocks in the linearized AWM have been demeaned and linearly de-trended whenever a linear trend was significant. In order to loose the impact of starting the expectations and lags initially in steady state (for example, interest rates on government debt build on 47 lags of the long-term rate), we discard the first 50 quarters of the shock sequences filtered under a white noise assumption and define the covariance matrix of shocks by its empirical counterpart, as such estimated from 1992q3 to 2000q4. We used the Clarida et al. (1999) rule with annual inflation rates to solve the model. This gave the best results among the four fixed rules discussed above in terms of white noise properties of the filtered shock sequences. First-order auto-correlation is significant for the employment shock, and the shock to long-term interest rates. For the latter shock,

${ }^{23}$ Here, we have eliminated the most obvious redundancies by substituting out some model variables. A few more could have been eliminated that way, however, at the cost of making the code less accessible for later changes to some of the structural equations. In addition, in contrast to Fagan et al. (2001), we have eliminated recursive equations defining variables which are not states of the economy (as an example, the HICP equation is derived from various other inflation terms but does not feed back anywhere into the model) and which are hence not relevant for our analysis.

${ }^{24}$ Again, a few of these lags could be substituted for at the cost of more complicated notation.

25 The model features a monetary policy shock, shocks to employment, the wage rate, the GDP deflator, the CPI, investment, price for capital goods, import and export prices. In addition, there are shocks to private consumption, stocks, exports, imports and a term structure shock. We also experimented with adding an empirical foreign interest rate shock as well adding shocks to the exogenous variables (excluding trend productivity and population growth); the findings of this study appeared to be robust to our choice of shocks. 
also second order serial correlation is significantly different from zero. We nevertheless maintain the white-noise assumption. ${ }^{26}$

\section{Calculation of Optimal Policy}

For the simple optimal policy rules, we proceed along the lines described in Levin et al. (2001). Namely, we start with an initial guess for the parameters of the monetary feedback rule, solve the model using the AIM programs of Anderson and Moore (1985), an implementation of the Blanchard and Kahn (1980) methodology, in order to obtain the rational expectations solution $y_{t}=A y_{t-1}+B u_{t}$, where $A$ is a stable matrix and $u$ is a white noise vector of shocks. Stationarity implies $V(y)=A V(y) Y^{\prime}+B V(u) B^{\prime}$, on which we iterate using the doubling algorithm described in Hansen and Sargent (1997). Using the constrained optimization routine fmincon in MATLAB 6 to minimize the loss-function, we set the convergence conditions to 1.e-6. In order to exclude any dependence on starting values, we start the optimization using three different plausible starting values. Results, however, did not appear to be sensitive to the starting values.

For the optimal policy under discretion in our quadratic framework, we apply the algorithms of Dennis (2001). While for smaller models Dennis' method for computing discretionary policy is robust to the starting values chosen, for the AWM the algorithm did not lead to convergence for arbitrary starting values (although they complied to stability and rank conditions laid out in his paper). Instead, we base our initial values on the respective blocks of the companion matrix of the model solution based on a fixed Taylor rule. ${ }^{27}$

\section{E Upper Bound on $\sigma_{\Delta r}$ for Discretion}

Let $V(x ; \lambda, \gamma)$ mark the variance for variable $x$ that results when minimizing loss function $L(\lambda, \gamma)$ in (1) using discretionary policy. Furthermore, let $\tilde{L}(\lambda, \gamma)=V(\pi ; \lambda, \gamma)+$ $\lambda V(y ; \lambda, \gamma)$ be the same loss function but disregarding $V(\Delta r ; \lambda, \gamma)$. In this setup, we have that $\frac{\partial V(\Delta r ; \lambda, \gamma)}{\partial \gamma}<0$ and $\frac{\partial \tilde{L}}{\partial \gamma}>0$.

We seek the minimum loss $\tilde{L}$ such that the bound on $\sigma_{\Delta r}$ is satisfied. For a specific $\lambda$, the figures reported in the two upper blocks of Table 5 therefore are obtained by mini-

${ }^{26}$ Again, the key results of the paper do not appear to be sensitive to this choice. We also experimented with single-equation shocks extracted from the non-linear model and redefined to be consistent with the definition of shocks the linear model entertains. Running SUR estimation on the system of shocks in order to capture joint properties of the time-series and taking into account up to second order serial correlation for each shock, we arrived at very similar conclusions for optimal policy.

27 Namely, we apply Taylor's (1993) rule to obtain the starting values. Experimenting with the other fixed rules mentioned in this study did not show any improvement in computational speed and neither did the optimal discretionary policy derived appear to be sensitive to the specific rule used to generate starting values - in line with theory. 
mizing $\gamma$ subject to $\sigma_{\Delta r}(\lambda, \gamma) \leq \bar{\sigma}$. The minimization is implemented by a line search over $\gamma$. At each step of the line search the outcome for discretionary policy when minimizing $L(\lambda, \gamma)$ is computed and it is evaluated whether $\sigma_{\Delta r}(\lambda, \gamma) \leq \bar{\sigma}$. Losses reported in Table 5 pertain to $\tilde{L}\left(\lambda, \gamma^{*}\right)$, where $\gamma^{*}$ is the optimal value of $\gamma$ from the line search.

\section{F Optimal Commitment}

As regards the computation of commitment policy, the AWM even after linearization is nearly non-stationary due to the "exogenous" processes - impeding the solution by AIM once costates are added. We resorted initially to Sims' (2001) procedure to identify the transition of structural shocks. In section, 4.2 however, we illustrate that even very complicated rules under commitment do not go a long way in achieving the sizeable gains under this identification scheme. To us these results indicate that the identification, in fact, may have a great bearing on the policy conclusions. We therefore include the commitment results in Table 11 for the sake of completeness only - but they should be treated with caution. We summarize the results for the cases with a flexible penalty for interest rate change variability, $\gamma>0 .{ }^{28}$ These commitment losses are somewhere between $50 \%$ and $82 \%$ of the discretionary losses.

\section{G Sensitivity analysis of results}

All in all, the results reported in the paper are dependent on the specification of the model used. As mentioned previously, the AWM model has a substantial degree of intrinsic (not induced by policy) persistence with long-lasting effects on output and inflation in the monetary transmission mechanism. In view of this, it seems warranted to conduct some further experiments, involving changes to the model employed. In this respect, it is interesting to explore how much results are sensitive to this feature of the model for plausible variants in some key equations. Firstly, the introduction of forward-lookingness in the wage and price setting block seems appealing. ${ }^{29}$ Secondly, given the importance of the exchange rate channel in the AWM, two alternative specifications were tested, one with a more forward-looking UIP, and one with contemporaneous UIP. These experiments give a first indication of the extent that the results are dependent on the degree of intrinsic persistence in the model. They were done for optimized policy under the contemporaneous rule with no restrictions on $\bar{\sigma}_{\Delta r}$. The results reported in the table 12 show that there seems

\footnotetext{
28 While in principle, a line search could be done to find a punishment $\gamma$ such that a fixed upper bound $\bar{\sigma}_{\Delta r}$ is met, using Sims' (2001) solver this proves rather burdensome in terms of computing time and would add little information.

${ }^{29}$ More precisely, the proxy for the expectation term has been made forward-looking by 4 quarters in both the wage and the output price equations - in line with Dieppe and Henry (2004)
} 
Table 11: Results for Optimal Commitment Policy under Sims' Identification ${ }^{a}$

\begin{tabular}{||c|c||c|c|c|c||c||}
\hline \hline$\lambda$ & $\gamma$ & $\sigma_{\text {gap }}$ & $\sigma_{\pi}$ & $\sigma_{\Delta r}$ & $\sigma_{r}$ & Loss \\
\hline \hline \multicolumn{7}{|c||}{$\bar{\sigma}_{\Delta r}$ free } \\
\hline 0.00 & 0.01 & 1.79 & 0.68 & 1.68 & 4.70 & 0.49 \\
\hline 0.10 & 0.01 & 1.09 & 0.71 & 2.16 & 4.91 & 0.67 \\
\hline 0.33 & 0.01 & 0.77 & 0.75 & 3.10 & 5.82 & 0.86 \\
\hline 1.00 & 0.01 & 0.55 & 0.80 & 4.33 & 6.69 & 1.13 \\
\hline 3.00 & 0.01 & 0.41 & 0.84 & 5.91 & 7.47 & 1.56 \\
\hline \hline \multicolumn{7}{|c||}{$\bar{\sigma}_{\Delta r}$ free } \\
\hline 0.00 & 0.10 & 1.72 & 0.73 & 0.74 & 3.18 & 0.58 \\
\hline 0.10 & 0.10 & 1.41 & 0.73 & 0.91 & 3.36 & 0.82 \\
\hline 0.33 & 0.10 & 1.13 & 0.76 & 1.34 & 3.97 & 1.18 \\
\hline 1.00 & 0.10 & 0.88 & 0.80 & 2.05 & 4.86 & 1.82 \\
\hline 3.00 & 0.10 & 0.67 & 0.84 & 3.02 & 5.81 & 2.95 \\
\hline \hline \multicolumn{7}{|c||}{$\bar{\sigma}_{\Delta r}$ free } \\
\hline 0.00 & 0.50 & 1.76 & 0.78 & 0.41 & 2.37 & 0.69 \\
\hline 0.10 & 0.50 & 1.60 & 0.78 & 0.47 & 2.43 & 0.97 \\
\hline 0.33 & 0.50 & 1.40 & 0.80 & 0.63 & 2.71 & 1.49 \\
\hline 1.00 & 0.50 & 1.17 & 0.85 & 0.98 & 3.34 & 2.57 \\
\hline 3.00 & 0.50 & 0.94 & 0.93 & 1.55 & 4.32 & 4.70 \\
\hline \hline \multicolumn{7}{|c||}{$\bar{\sigma}_{\Delta r}$ free } \\
\hline 0.00 & 1.00 & 1.79 & 0.81 & 0.31 & 2.03 & 0.76 \\
\hline 0.10 & 1.00 & 1.66 & 0.81 & 0.34 & 2.05 & 1.05 \\
\hline 0.33 & 1.00 & 1.50 & 0.83 & 0.43 & 2.23 & 1.63 \\
\hline 1.00 & 1.00 & 1.28 & 0.89 & 0.67 & 2.76 & 2.89 \\
\hline 3.00 & 1.00 & 1.05 & 1.00 & 1.10 & 3.73 & 5.53 \\
\hline \hline
\end{tabular}

${ }^{a}$ Optimal commitment policy using the identification scheme of Sims (2001).

to be minimal sensitivity to increasing the forward-lookingness, but there is some change in the $\beta$ parameter when the UIP is made contemporaneous. While this analysis illustrates

Table 12: Sensitivity of results ${ }^{a}$

\begin{tabular}{|c|c|c|c|c|c|c|}
\hline & $\overline{\bar{\lambda}}$ & $\gamma$ & $\rho$ & $\alpha$ & $\overline{\bar{\beta}}$ & 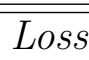 \\
\hline \multicolumn{7}{|c|}{$\bar{\sigma}_{\Delta r}$ free } \\
\hline base & 1.00 & 0.50 & 0.46 & 0.25 & 1.68 & 3.88 \\
\hline yfd forward 4 & 1.00 & 0.50 & 0.44 & 0.25 & 1.78 & 4.02 \\
\hline wrn forward 4 & 1.00 & 0.50 & 0.46 & 0.25 & 1.67 & 3.84 \\
\hline een forward4 & 1.00 & 0.50 & 0.38 & 0.24 & 1.77 & 3.81 \\
\hline een forward0 & 1.00 & 0.50 & 0.40 & 0.07 & 1.82 & 4.20 \\
\hline
\end{tabular}

${ }^{a}$ The Table reports results for changing the forward-lookingness of the model where yfd is the GDP at factor cost equation, wrn is the wage rate equation, and een is the UIP equation. 
that the small perturbations of the model around the structure employed in the paper do not change the quantitative results much, comfort is limited. Most likely would larger deviations in the direction of more forward-lookingness have a bearing on the results as the body of existing literature suggests. More work needs to be done in this area - which we leave for future research. 


\section{European Central Bank working paper series}

For a complete list of Working Papers published by the ECB, please visit the ECB's website (http://www.ecb.int).

302 “Deposit insurance, moral hazard and market monitoring” by R. Gropp and J. Vesala, February 2004.

303 "Fiscal policy events and interest rate swap spreads: evidence from the EU" by A. Afonso and R. Strauch, February 2004.

304 "Equilibrium unemployment, job flows and inflation dynamics" by A. Trigari, February 2004.

305 "A structural common factor approach to core inflation estimation and forecasting" by C. Morana, February 2004.

306 "A markup model of inflation for the euro area" by C. Bowdler and E. S. Jansen, February 2004.

307 "Budgetary forecasts in Europe - the track record of stability and convergence programmes" by R. Strauch, M. Hallerberg and J. von Hagen, February 2004.

308 "International risk-sharing and the transmission of productivity shocks" by G. Corsetti, L. Dedola and S. Leduc, February 2004.

309 "Monetary policy shocks in the euro area and global liquidity spillovers" by J. Sousa and A. Zaghini, February 2004.

310 "International equity flows and returns: A quantitative equilibrium approach" by R. Albuquerque, G. H. Bauer and M. Schneider, February 2004.

311 "Current account dynamics in OECD and EU acceding countries - an intertemporal approach" by M. Bussière, M. Fratzscher and G. Müller, February 2004.

312 "Similarities and convergence in G-7 cycles" by F. Canova, M. Ciccarelli and E. Ortega, February 2004.

313 "The high-yield segment of the corporate bond market: a diffusion modelling approach for the United States, the United Kingdom and the euro area" by G. de Bondt and D. Marqués, February 2004.

314 "Exchange rate risks and asset prices in a small open economy" by A. Derviz, March 2004.

315 "Option-implied asymmetries in bond market expectations around monetary policy actions of the ECB" by S. Vähämaa, March 2004. 
316 “Cooperation in international banking supervision” by C. Holthausen and T. Rønde, March 2004.

317 "Fiscal policy and inflation volatility" by P. C. Rother, March 2004.

318 "Gross job flows and institutions in Europe" by R. Gómez-Salvador, J. Messina and G. Vallanti, March 2004.

319 "Risk sharing through financial markets with endogenous enforcement of trades” by T. V. Köppl, March 2004.

320 "Institutions and service employment: a panel study for OECD countries" by J. Messina, March 2004.

321 "Frequency domain principal components estimation of fractionally cointegrated processes" by C. Morana, March 2004.

322 "Modelling inflation in the euro area" by E. S. Jansen, March 2004.

323 "On the indeterminacy of New-Keynesian economics" by A. Beyer and R. E. A. Farmer, March 2004.

324 "Fundamentals and joint currency crises” by P. Hartmann, S. Straetmans and C. G. de Vries, March 2004.

325 "What are the spill-overs from fiscal shocks in Europe? An empirical analysis" by M. Giuliodori and R. Beetsma, March 2004.

326 "The great depression and the Friedman-Schwartz hypothesis" by L. Christiano, R. Motto and M. Rostagno, March 2004.

327 "Diversification in euro area stock markets: country versus industry" by G. A. Moerman, April 2004.

328 "Non-fundamental exchange rate volatility and welfare” by R. Straub and I. Tchakarov, April 2004.

329 "On the determinants of euro area FDI to the United States: the knowledge-capital-Tobin's Q framework, by R. A. De Santis, R. Anderton and A. Hijzen, April 2004.

330 "The demand for euro area currencies: past, present and future" by B. Fischer, P. Köhler and F. Seitz, April 2004.

33I "How frequently do prices change? evidence based on the micro data underlying the Belgian CPI" by L. Aucremanne and E. Dhyne, April 2004.

332 "Stylised features of price setting behaviour in Portugal: 1992-200I" by M. Dias, D. Dias and P. D. Neves, April 2004.

333 "The pricing behaviour of Italian firms: New survey evidence on price stickiness" by S. Fabiani, A. Gattulli and R. Sabbatini, April 2004.

334 "Is inflation persistence intrinsic in industrial economies?" by A. T. Levin and J. M. Piger, April 2004.

335 “Has eura-area inflation persistence changed over time?” by G. O’Reilly and K. Whelan, April 2004.

336 "The great inflation of the 1970s" by F. Collard and H. Dellas, April 2004.

337 "The decline of activist stabilization policy: Natural rate misperceptions, learning and expectations" by A. Orphanides and J. C. Williams, April 2004. 
338 "The optimal degree of discretion in monetary policy" by S. Athey, A. Atkeson and P. J. Kehoe, April 2004.

339 "Understanding the effects of government spending on consumption" by J. Galí, J. D. López-Salido and J. Vallés, April 2004.

340 "Indeterminacy with inflation-forecast-based rules in a two-bloc model" by N. Batini, P.Levine and J. Pearlman, April 2004.

34 I "Benefits and spillovers of greater competition in Europe: A macroeconomic assessment" by T. Bayoumi, D. Laxton and P. Pesenti, April 2004.

342 "Equal size, equal role? Interest rate interdependence between the euro area and the United States" by M. Ehrmann and M. Fratzscher, April 2004.

343 "Monetary discretion, pricing complementarity and dynamic multiple equilibria” by R. G. King and A. L. Wolman, April 2004.

344 "Ramsey monetary policy and international relative prices" by E. Faia and T. Monacelli, April 2004.

345 “Optimal monetary and fiscal policy: A linear-quadratic approach” by P. Benigno and M. Woodford, April 2004.

346 "Perpetual youth and endogenous labour supply: a problem and a possible solution" by G. Ascari and N. Rankin, April 2004.

347 "Firms' investment decisions in response to demand and price uncertainty" by C. Fuss and P. Vermeulen, April 2004.

348 "Financial openness and growth: Short-run gain, long-run pain?" by M. Fratzscher and M. Bussiere, April 2004.

349 "Estimating the rank of the spectral density matrix" by G. Camba-Mendez and G. Kapetanios, April 2004.

350 "Exchange-rate policy and the zero bound on nominal interest rates" by G. Camba-Mendez and G. Kapetanios, April 2004.

$35 \mathrm{I}$ "Interest rate determination in the interbank market" by V. Gaspar, G. P. Quirós and H. R. Mendizábal, April 2004.

352 "Forecasting inflation with thick models and neural networks" by P. McNelis and P. McAdam, April 2004.

353 "Towards the estimation of equilibrium exchange rates for CEE acceding countries: methodological issues and a panel cointegration perspective” by F. Maeso-Fernandez, C. Osbat and B. Schnatz, April 2004.

354 "Taking stock: monetary policy transmission to equity markets" by M. Ehrmann and M. Fratzscher, May 2004.

355 "Production interdependence and welfare" by K. X. D. Huang and Z. Liu, May 2004. 
356 "Developing a euro area accounting matrix: issues and applications" by T. Jellema, S. Keuning, P. McAdam and R. Mink, May 2004.

357 "Seasonal adjustment and the detection of business cycle phases" by A. M. Mir, and D. R. Osborn, May 2004.

358 "Did the pattern of aggregate employment growth change in the euro area in the late 1990s?" by G. Mourre, May 2004.

359 “The longer term refinancing operations of the ECB” by T. Linzert, D. Nautz and U. Bindseil, May 2004.

360 "Optimal monetary policy rules for the euro area: an analysis using the area wide model" by A. Dieppe, K. Küster and P. McAdam, May 2004. 
